\begin{tabular}{|c|c|c|c|c|}
\hline (2) & Volume 25 & $\begin{array}{l}\text { PORT SAID ENGINEERING JOURNAL } \\
\text { Faculty of Engineering - Port Said University } \\
\text { No. } 2 \text { September } 2021 \\
\text {-Civil Engineering- }\end{array}$ & pp. $60-79$ & \\
\hline
\end{tabular}

\title{
Assessment of the Finite Element Analysis of Portal Steel Frames with Cold Formed Rectangular Hollow Sections Including Imperfections and Residual Stresses
}

\author{
Tarek Sharaf ${ }^{1}$, Sarah Elglaad ${ }^{2}$, Mohamed ElGhandour $^{3}$, Ashraf ElSabbagh $^{4}$
}

\begin{abstract}
This paper aims to assess the finite element analysis in predicting the behavior of portal steel frames fabricated from cold formed steel (CFS) rectangular hollow sections. ABAQUS ${ }^{\circledR}$ was used in the current research to discuss and validate the finite element models with experimental tests on portal frames and knee joints of portal frames. The influence of the nonlinear finite element solution methods, loading type controls, initial imperfections in CFS, residual stresses, mesh density, and different element types were studied. Also, the method of representing the initial imperfection were considered and found to have significant influence on the results. These considerations enabled performing an extensive parametric study. The parametric study consisted of 144 portal frames with various dimensions and RHS sizes. The ultimate load capacity as well as horizontal and vertical displacements that represent the nonlinear behavior of such steel frames were obtained. The failure modes due to material yielding, local buckling, plastic hinges formation as well as interaction of material yielding, and local buckling were also recorded.
\end{abstract}

Keywords: Cold formed hollow sections, Steel frames, Knee joints, Finite element analysis, Initial imperfections, Residual stresses, Failure Modes.

\section{INTRODUCTION}

Cold formed sections have already been used in steel structures providing various benefits to a range of construction and mechanical applications which include portal frames in a large scale that are consisted of open and closed sections. For most common cold formed opened sections, channels attached back-to-back and Z-section members were chosen to design the steel frames by many researchers. Cold-formed tubes are available in many forms such as: rectangular hollow sections (RHS), square hollow sections (SHS), circular hollow sections (CHS), and oval hollow section (OHS). Androić et al. [1] performed a

\footnotetext{
${ }^{1}$ Associate Professor Civil Engineering Department, Faculty of Engineering, Port Said University, Port Said, Egypt, email: tarek_sharaf@eng.psu.edu.eg

${ }^{2}$ Researcher, Civil Engineering Department, Faculty of Engineering, Port Said University, Port Said, Egypt email: elglaad10@gmail.com.

${ }^{3}$ Professor Civil Engineering Department, Faculty of Engineering, Port Said University, Port Said, Egypt, email :dr.elghandor@gmail.com

${ }^{4}$ Associate Professor Civil Engineering Department, Faculty of Engineering, Port Said University, Port Said, Egypt, email:ashraf_ims@yahoo.com
}

Received: 05-05-2021, Accepted: 12-07-2021

DOI $\underline{\text { 10.21608/PSERJ.2021.75314.1111 }}$ comparison between cold formed and hot finished hollow sections and it was shown from the comparison that the manufactured cold-formed hollow sections according to EN 10219 [39] are appropriate for all types of steel structures. Several researches studied the new sections used in steel structure construction applications such as oval hollow sections. Zhu and Young [36] tested 28 columns that were fabricated from cold formed steel oval hollow sections (OHS) subjected to axial compression between fixed ends. The study evaluated the failure modes such as flexural buckling, local buckling, and material yielding. Lie et al. [2] studied the behavior of cold formed rectangular steel thickwalled columns under either axial or eccentric compression using both experimental tests and FEA including the effects of residual stresses and initial imperfection. The results show that direct loaded cold-formed rectangular steel columns have higher cold-formed effects than indirect cold-formed rectangular steel columns and the higher bearing capacity for cold formed rectangular steel columns was under axial loads. Different failure modes (overall buckling or local buckling) occur for cold formed hollow sections due to different slenderness ratios and width-to-thickness ratios [3] and local buckling mainly occurred at the welded parts that leads to failure in welding [4-5]. Wilkinson [6] used the finite element analysis to investigate trends and the occurrence of inelastic instability in cold formed RHS beams. The study illustrated that there are three main types of residual stresses for RHS; membrane residual stresses, bending residual stresses, and layering residual stresses, however bending 
residual stresses and layering residual stresses were used in FEA models in based on Key [37]. Wilkinson and Hancock [7] provided tests on various types of knee joints for steel portal frames constructed from cold-formed rectangular hollow sections (RHS) to study the behavior of the connections to form plastic hinges. Using the ANSYS nonlinear finite element analysis programme, EL-Ghandour explored alternative approaches for stiffening various types of knee joints for steel portal frames produced from coldformed rectangular hollow sections (RHS) to investigate the ability of the connections to form plastic hinges [40]. Following the approach in [41], V.Priyadarshinil et al studied experimentally the behavior of cold formed steel frames using hollow section which connected along major and minor axes connections. It resulted in the load carrying capacity of frames connected along the major axis is found to be 38 percent higher than that of frames connected along the minor axis. Also the deflection of the minor axis connected frame was found to be 6.06 percent higher than that of the main axis connected frame, so major axis connected frames work better than minor axis connected frames. Wilkinson and Hancock [8] studied three large scale portal frames manufactured from cold-formed RHS in both Grade C350 and Grade C450 steel. The results showed that each frame formed a plastic collapse mechanism. Mang et al. [9] studied the static behavior of knee joints made from circular hollow sections (CHS). Puthli et al. [10] studied the fatigue behavior of knee connections in both RHS and CHS. Kwon et al. [11] provided portal frames which consisted of closed cold-formed steel PRY sections to investigate the compressive and the flexural strengths of the PRY sections, the structural behavior of the connections including the moment-rotation relation, and the yield and the ultimate moment capacities of the connections. The connection test results were compared with those achieved by using advanced analysis procedures. Other researchers [12-15] performed studies on the effective moment framing in coldformed steel structures to employ cold-formed steel $\mathrm{C}$ sections with bolted moment connections as primary structural members in low to medium rise buildings as well as in portal frames with medium spans. Chung et al. [16] and Darcy and Mahendran [17] studied bolted moment connections in portal frames. While other researchers [1822] presented the study of stiffened and unstiffened knee connections for portal frames with CHS and RHS members. Mahendran and Avery [23] and Avery [24] used the experimental and the FEA to analyze steel frame structures comprising compact and non-compact sections, residual stresses and local buckling were studied as well. Other studies [25-27] proposed the viability to construct portal frames fabricated from cold formed sections with spans up to $12 \mathrm{~m}$ which interested with eaves and apex joints to be rigid. Kurobane [28] experimentally evaluated the behavior of truss structures with tube-to-tube connections.

The current research aims to evaluate the use of the finite element analysis to figure out the behavior of cold formed steel structures as well as to determine which finite element is more suitable to be used in the analysis. Also, the effect of both residual stresses and initial imperfections modeled by the structural mode shapes and which mode shape should be used by the designer to analyze such structures.

\section{FINITE ELEMENT MODELLING}

Finite element models were created for comparison with experimental tests results to investigate the behavior of steel frame studied by Avery [24] and various types of knee joints of portal frames fabricated from CFHS studied by Wilkinson and Hancock [29]. This section aims to study a series of finite element models with the effect of different inputs such as initial imperfection and residual stresses as well as the effect of mode shapes and then compare them with the experimental results.

\subsection{Frame Finite Element Model}

The frame model consists of cold-formed rectangular hollow sections $(200 \times 100 \times 4$ RHS $)$. The frame span was 4.0 $\mathrm{m}$ between column center lines while the height was $3.0 \mathrm{~m}$. Minor axis bending was restrained to exclude out-of-plane column buckling, Avery [24]. Both rectangular hollow sections of the beam and the column were connected using welding; connections were modelled with appropriate contact in ABAQUS ${ }^{\circledR}$. The floor girder and the two-column base plates, shown in Figure 1, were modeled as a long thick plate with a thickness of $25 \mathrm{~mm}$ that was welded directly to the ends of the two columns, as shown in Figure 2, to act as a floor girder which rested on roller bearings boundary to be free and move laterally in-plane as shown in Figure 1.

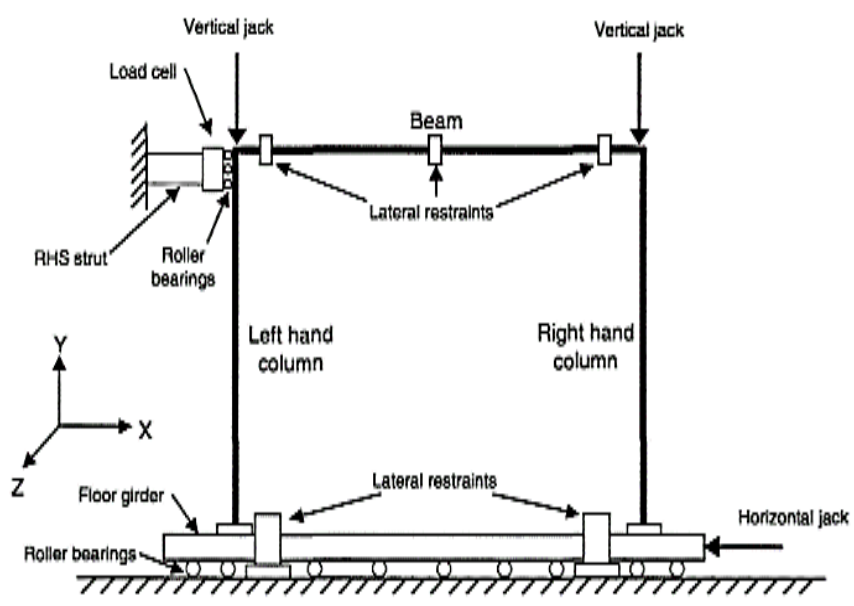

Figure 1:Test experiment and loading arrangement Avery [24] 


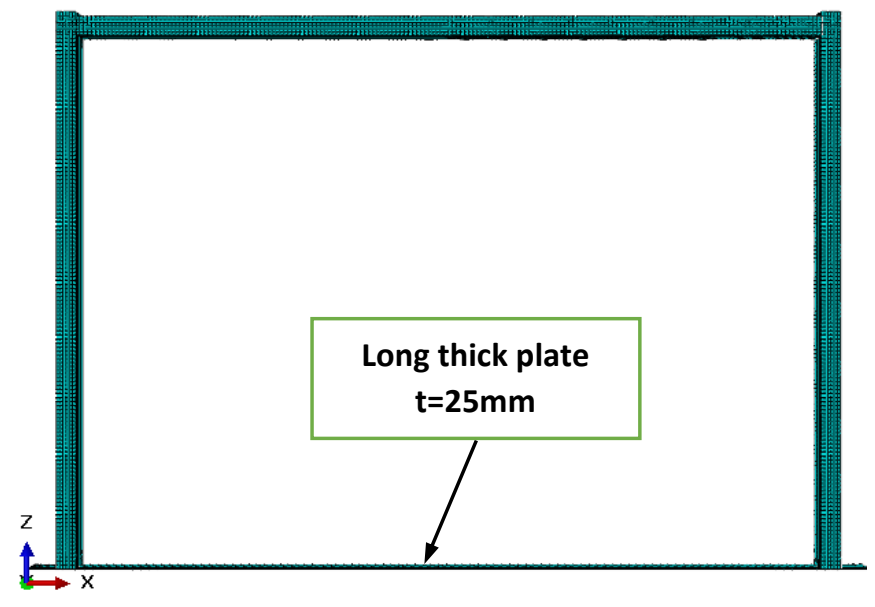

Figure 2: The three-dimensional finite element model of frame with long thick base plate

\subsubsection{Nonlinear Solution}

The standard static general method (the Newton-Raphson method) was used to model the frame with maximum number of load increments $=100$, initial increment size $=$ 0.05 , maximum increment size $=0.05$ and minimum increment size $=0.000001[24]$.

\subsubsection{Material Modeling}

Young's modulus, Poisson's ratio, and material nonlinearity were taken into consideration in the finite element analysis. It was essential to input the material accurately in the finite element analysis as it significantly affects the results, so it must follow the multi-linear stressstrain curves obtained from tensile testing of the $200 \times 100 \times 4$ RHS flange and web samples grade 350 steel that were presented in Avery [24]. The average of each of the two specimens of flange and web was taken for use in the finite element analysis model. Equations (1) and (2) [38] were used to convert stress-strain data conducted from tensile test to true stress and logarithmic plastic strain. Furthermore, the values resulting from the equations were entered into the FE program. The relation between true stress and true plastic strain is shown in Figure (3). Linear elastic material elasticity modulus and Poisson's ratio were $\mathrm{E}=200000 \mathrm{MPa}, v=0.3$, respectively.

$$
\begin{aligned}
& \sigma_{\text {true }}=\sigma_{\text {nom }}\left(1+\varepsilon_{\text {nom }}\right) \\
& \varepsilon_{p(\ln )}=\ln \left(1+\varepsilon_{\text {nom }}\right)-\frac{\sigma_{\text {true }}}{E}
\end{aligned}
$$

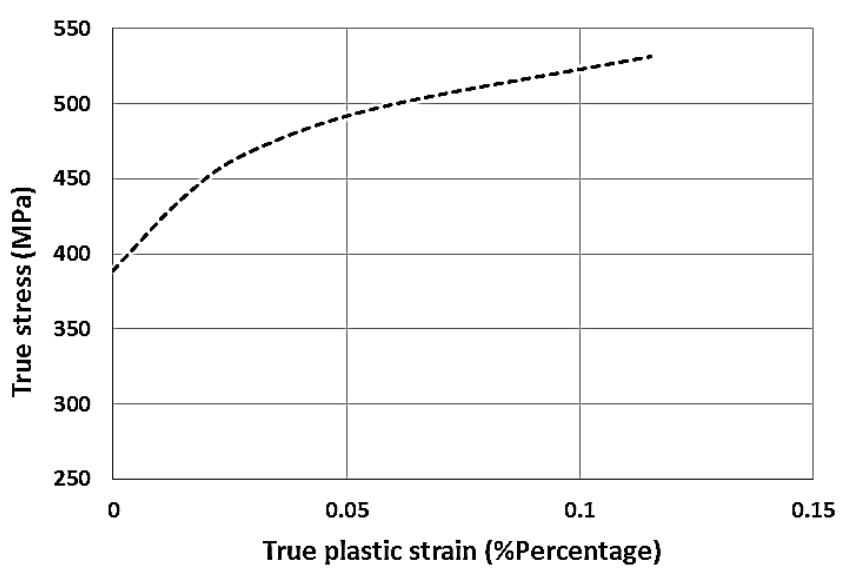

Figure 3: True stress-strain curve derived from a tensile coupon test for grade 350 steel [24]

\subsubsection{Element Type and Mesh Density}

Different shell elements are available in the finite element software. All the quadrilateral shell elements (S4, S4R, S4R5, and S8R5) are usually used to model thin structural elements which suites the analysis of thin-walled structure. It is necessary to obtain more accurate results and therefore, comparisons must be made between different densities and element types to estimate the sensitivity of the element. Four shell elements and three mesh densities (coarse $21 \times 21$ $\mathrm{mm} 2$, medium $14 \times 14 \mathrm{~mm} 2$, and fine $7 \times 7 \mathrm{~mm} 2$ ), as shown in Figure (4), were chosen in the current study to verify the frame in Avery [24].

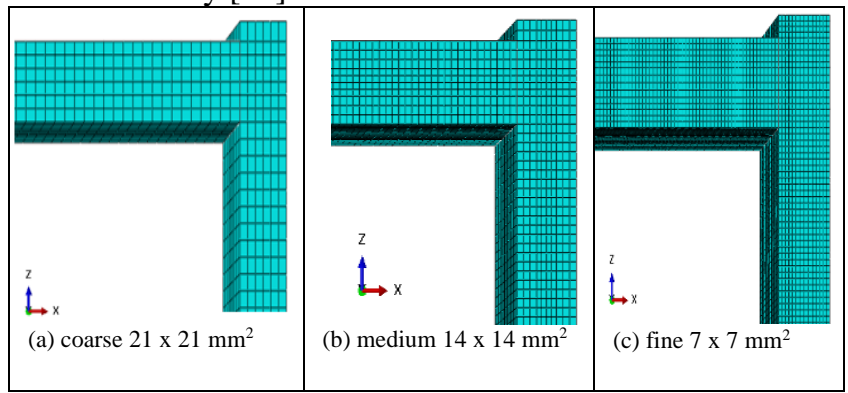

Figure 4:Three different finite element meshes for frame connection

Only the S4 element uses full integration while the rest of the elements use the reduced integration. Finite element types: S4, S4R and S4R5 use linear interpolation, but the element S8R5 generates a quadratic shape function. For firstorder shell elements which generate linear interpolation, S4 and S4R have 4-node doubly curved general-purpose thin/thick shell with six degrees of freedom at each node: three translational and three rotational. However, S4R5 has 4-node doubly thin curved shell only with five degrees of freedom at each node: three translational and two in-plane rotational. The second-order shell elements such as S8R5 has 8 -node doubly thin curved shell with five degrees of freedom per node. 


\subsubsection{Loads and Boundary Conditions}

The frame model was loaded using load control method which was applied as equal vertical loads at the center of the top of each column and horizontal load as concentrated force at a thick long plate adjacent to the base of the right-hand column to simulate vertical and horizontal jacks in the test [24]. Vertical loads were applied on the two reference points located at the centroids of the top of two columns, which are coupled with the surfaces of the cross-sections at both tops of frame columns. The vertical to horizontal load ratio was approximately 8:1 where the vertical load was approximately eight times the horizontal load. Loads were implemented simultaneously in the model. Translation DOF U1 was restrained at the top of the left column at reference point which was coupled with the associated nodes in the top of the left column.

To ensure that the long thick base plate which was free to move laterally in-plane was modelled to simulate the test, its boundary conditions, as indicated in Figure 5, were: translations $\mathrm{U} 1$ and $\mathrm{U} 2$ were free, $\mathrm{U} 3$ was restrained, and all rotations UR1, UR2, and UR3 were restrained. The frame test was full lateral restraint because the aim of the test is to investigate the influence of in-plane buckling and prevent lateral (out-of-plane) movement. Hence, the frame was restrained laterally, i.e., the DOF $\mathrm{U} 2$ was retrained, at five places to prevent the out-of-plane movement. Three was at the middle point and near end points while the other two were at the base plate near the two column bottoms, as shown in Figure 5, to simulate the lateral supports shown in Figure 1.

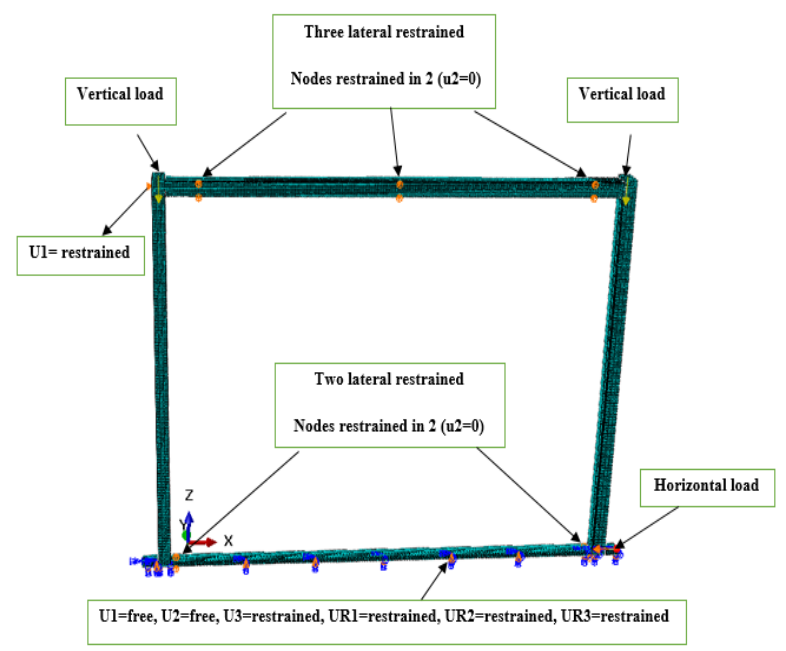

Figure 5: Finite element meshing, loading and boundary conditions for frame.

\subsubsection{Initial Geometric Imperfections}

It is known that initial geometric imperfections affect the thin-walled cold-formed members remarkably. Initial imperfection explicitly influences the frame strength where the ultimate load decreases and the ductility increases. In the present study, the magnitudes of the imperfections were taken as a portion of the plate thickness $(0.1 \mathrm{t}, 0.5 \mathrm{t}$, and $1.0 \mathrm{t})$ $[30,34,35]$.

Two steps have been implemented for the finite element analysis:

Firstly, linear elastic buckling analysis was carried out to obtain twelve mode shapes for the frame. Then, check all the modes to determine which mode shape should be used to generate the initial imperfections in the non-linear analysis.

Secondly, the non-linear analysis including both material nonlinearity and geometric nonlinearity, residual stresses (RS), and initial imperfections (IMP) was performed. Results were conducted for the finite element models with different values of initial imperfections (W-IMP) and without initial imperfections (Wo-IMP) to show the influence of imperfection on the frame and compare results with the experimental tests.

\subsubsection{Residual Stresses}

Longitudinal membrane, longitudinal bending, longitudinal layering, transverse bending, and transverse layering residual stress components were measured by Key and Hancock [31]. They indicated that there are two types of residual stresses which have the most important effect on cold-formed steel structure members, as shown in Figure (6), namely:

1- Longitudinal bending residual stresses.

2- Membrane residual stresses.

Residual stresses were simplified in Figure (6) based on Key and Hancock [31].

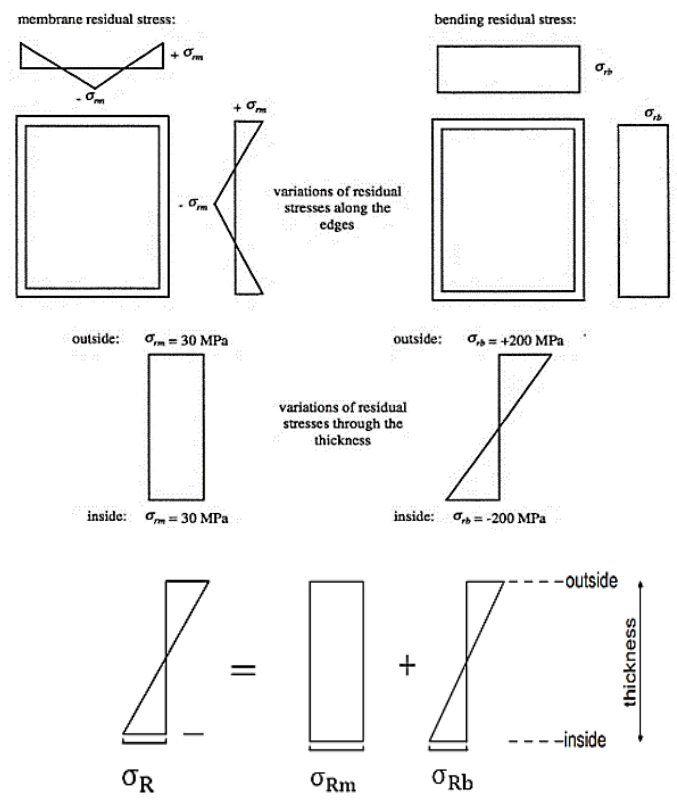

Figure 6: Longitudinal membrane and bending residual stress distributions for rectangular hollow sections (top) [31], Definition of bending and membrane residual stress (bottom)

The residual stresses were modelled in the predefined field outside and inside rectangular hollow sections as shown in 
Figure 6. To model the distribution of bending residual stress in cold-formed rectangular hollow sections, nine integration points through the thickness of the shell element were used instead of the default of five integration points [24].

\subsubsection{Results and Discussion:}

\subsubsection{Mesh Sensitivity Results:}

In order to deduce which element type was able to give more accurate results, the nonlinear finite element results for 4 models including linear and quadratic elements with medium mesh density were compared with experimental test results [24]. Figure 7 shows the relationship between the vertical load and the vertical displacement as well as the relationship between the horizontal load and the horizontal displacement. It is observed from Figure 7 that linear shell elements have great compatibility. That is, the results of the linear shell elements S4, S4R, and S4R5, are more accurate comparing with the test results. On the other hand, the results of quadratic shell elements have less accuracy comparing with the test results.
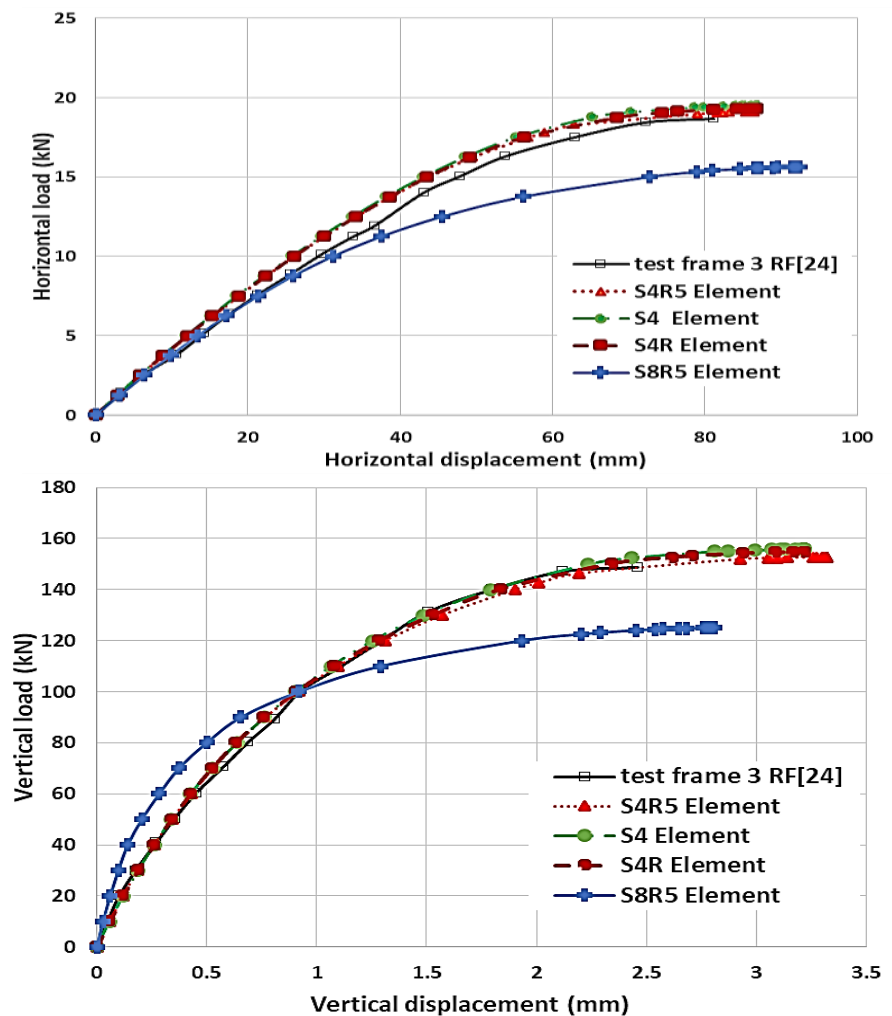

Figure 7: Comparison between models with different element types with linear and quadratic elements: Top) loadhorizontal displacement and bottom) Load-vertical displacement.

To improve the solution accuracy and significantly reduce run time so many shell element types use reduced (lower order) integration to form the element stiffness matrix. The S4 shell element is fully integrated and general-purpose and allows for transverse shear while the S4R5 element uses reduced integration and is needed in cases where transverse shear flexibility is negligible as for the present type of frames. Therefore, the S4R5 element was selected in the present study. Moreover, the results could be sensitive to mesh density, so that mesh sensitivity analysis was carried out. Figure 8 shows results of 3 models to illustrate comparison between finite element models with different mesh densities namely coarse, medium, and fine with constant value of imperfection (IMP $=0.5 \mathrm{t}$ ). Figure (8) shows the comparison between models using linear S4R5 element with varying mesh densities as coarse $21 \times 21 \mathrm{~mm} 2$, medium $14 \times 14 \mathrm{~mm} 2$, and fine mesh $7 \times 7 \mathrm{~mm} 2$. Medium mesh with $(14 \times 14 \mathrm{~mm} 2)$ has been selected in this research as it has considerable agreement with test results as shown in Figure (8) and less run time compared with fine mesh which needs more analysis time.
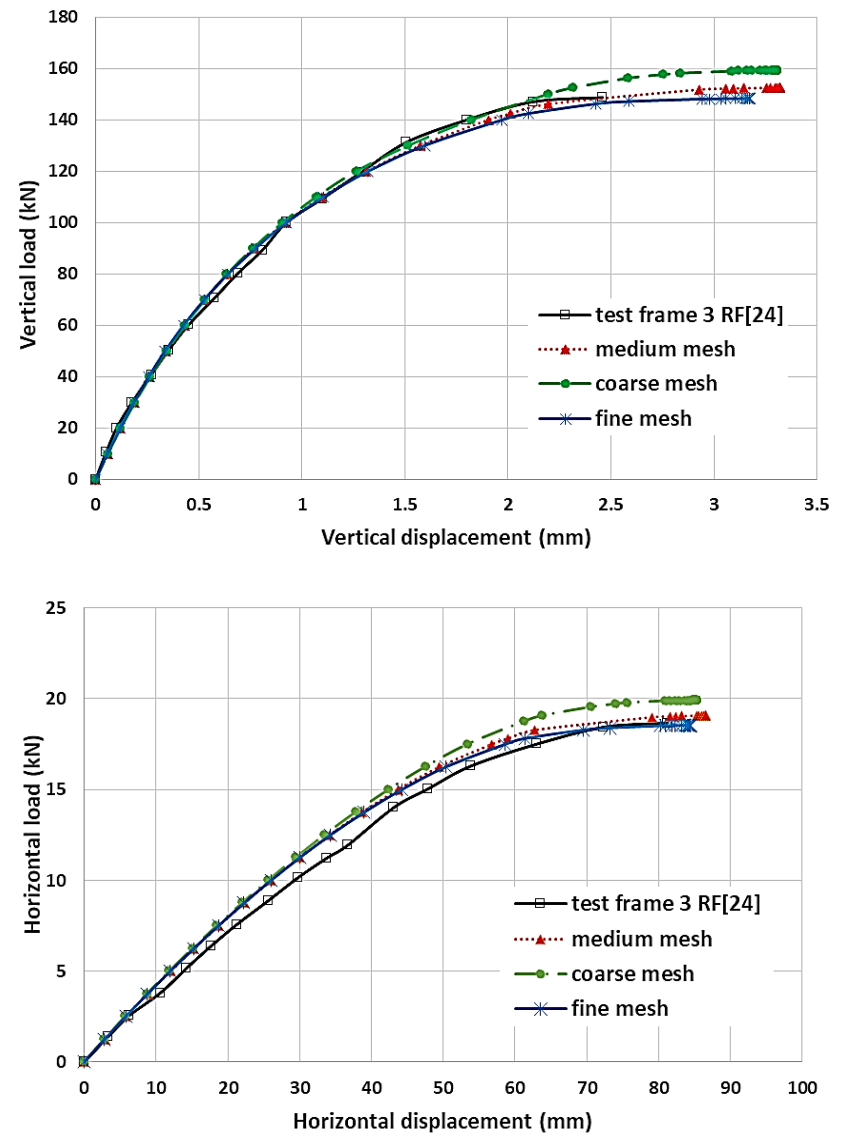

Figure 8: Mesh sensitivity analysis results

In the analysis, initial imperfection has been taken equal a percent of the thickness of $(0.1 \mathrm{t}, 0.5 \mathrm{t}$ and $1.0 \mathrm{t})$, respectively, with 12 buckling mode shapes assigned to each value of initial imperfection. Figure (9) shows a comparison between, for constant value of imperfection (IMP $=0.5 \mathrm{t}$ ), the vertical 
and horizontal load with the vertical and horizontal displacement, respectively.
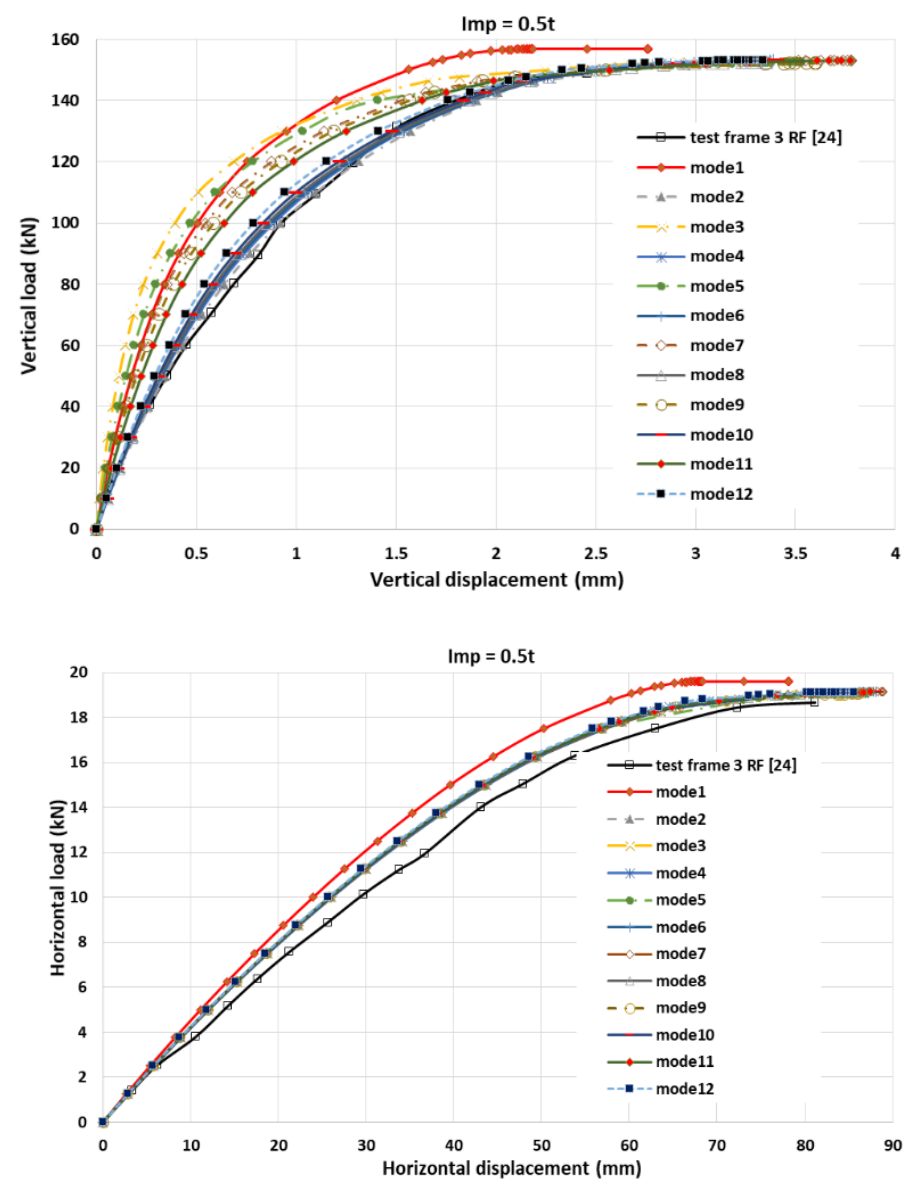

Figure 9: Load-displacement relations with value of imperfection ( $0.5 t)$ and it was analyzed using 12 buckling mode shapes.

It is realized that the second method of imperfection, 50 percent of the thickness, accompanied with the mode shape number 2, provide the most critical imperfection, and give the best results comparing with the test results The failed inplane instability, yielding material, and local buckling near the base of both columns and top of each column as shown in Figure (10).

Figure (11) shows the initial imperfections' sensitivity for the most suitable buckling mode (mode 2) using different imperfection magnitudes as a percentage of the thickness (0.1t, 0.5t, 1.0t).

The comparison shows good correlation between the test results and the results obtained using $0.5 \mathrm{t}$ imperfection with the second mode shape. Models with imperfection, without imperfection, with residual stresses, and without residual stresses were conducted as shown in Figure (12).

The figure 12 illustrates the importance of considering the effect of imperfections as well as residual stresses which remarkably influence the results of the non-linear analysis and ultimate load magnitude.

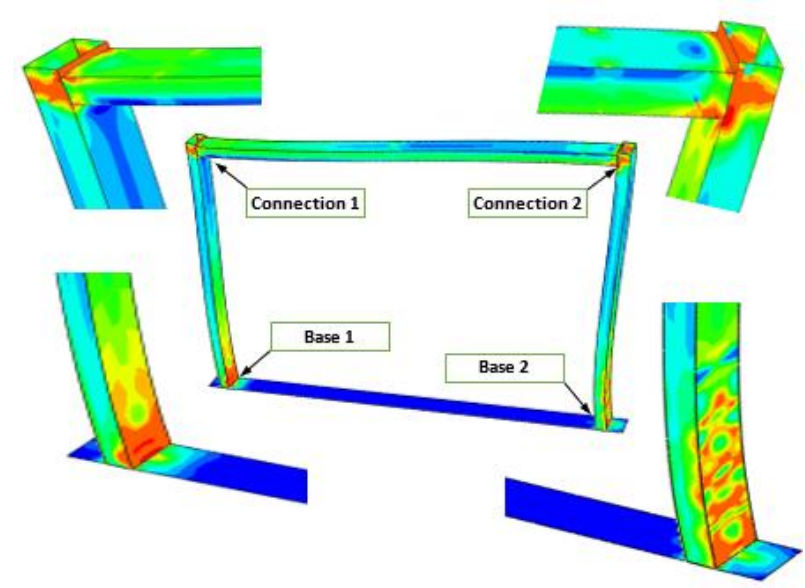

Figure 10: Failure of frame at ultimate load

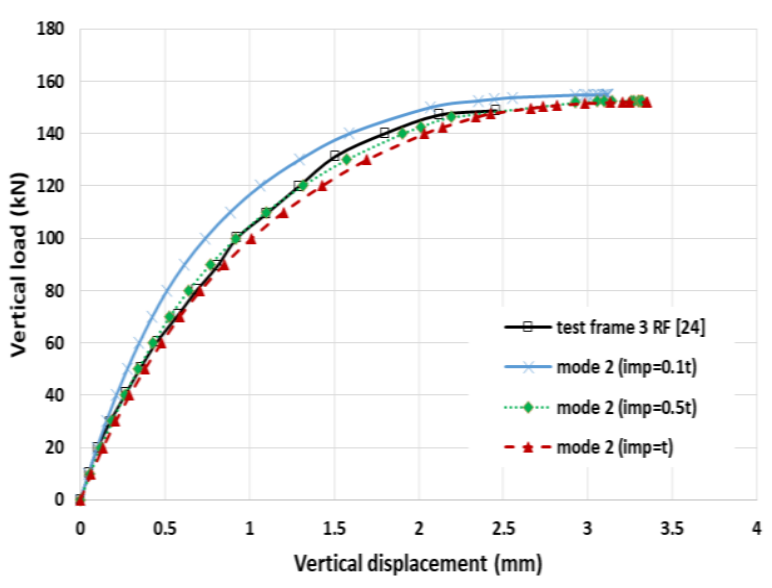

Figure 11: Typical vertical load versus vertical displacement curve to show sensitivity of the results to initial imperfections.

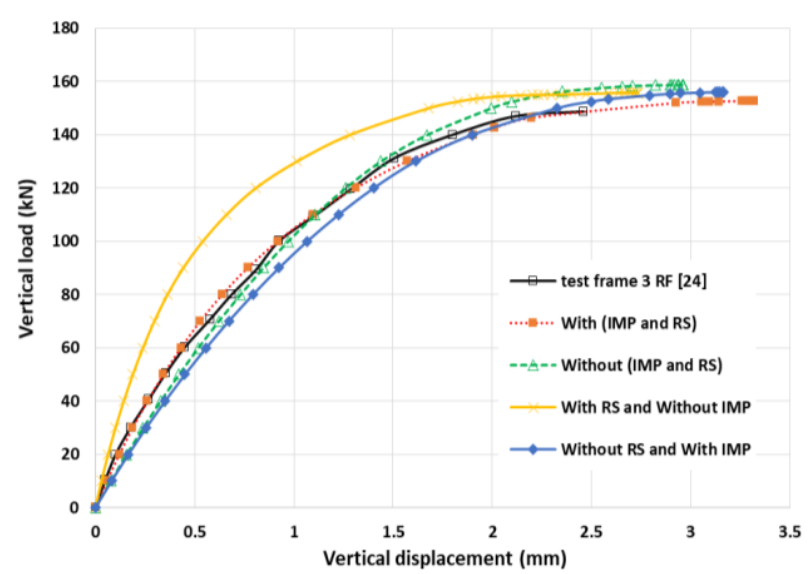

Figure 12: Typical vertical load versus vertical displacement curve to show the sensitivity of the results to both imperfections and residual stresses. 


\subsubsection{Comparison between Non-Linear Solutions}

A comparison was made between two methods of nonlinear analyzes namely static-general and static-riks with load control method. As can be seen from Figure (13), staticriks method can successfully predict the post-peak load and frame behavior after failure load more than static general method at which the peak resisting load of the frame was predicted then solution stopped. Both methods had a very good agreement with test results [24] in the pre-peak stage of analysis. However, static-general method was chosen wherever the post peak behavior is not required, as in [24] where the post-peak failure records were not listed.

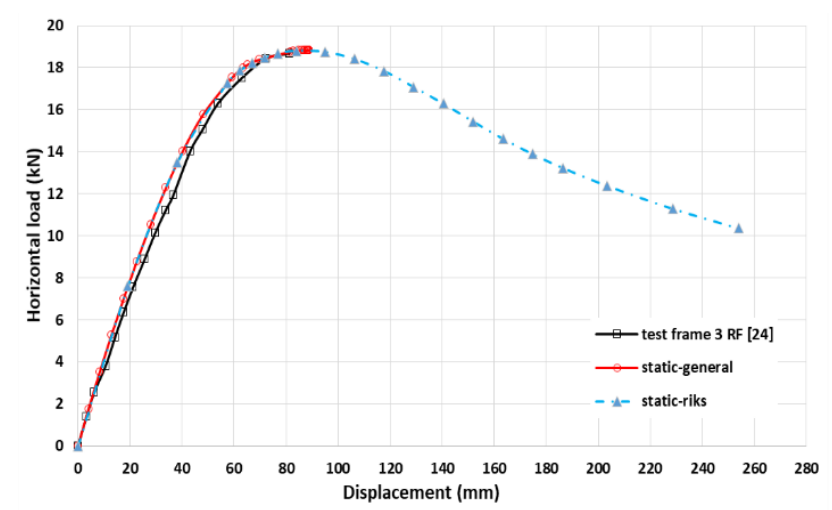

Figure 13: Typical horizontal load versus horizontal displacement curve to show comparison between non-linear analysis methods (static-general and static-riks).

\subsection{Knee Joints Finite Element Models}

This section demonstrates verification of experimental tests for three knee joints in a portal frame application which were introduced in Wilkinson and Hancock [29] and aimed to simulate the behavior of a knee joint in a portal frame which could be modelled using the finite element analysis as shown in Figure (14). All three knee joints consisted of coldformed rectangular hollow sections $(150 \times 50 \times 4$ RHS $)$ namely:

\section{a) Unstiffened welded connection (US)}

The unstiffened welded connection was modelled by cutting end of two rectangular CFHS at an angle of 52.5o to create a total of 105 o then two sections were welded directly with each other based on studies in $[32,33,29]$ as shown in Figure (14.a).

\section{b) Stiffened welded connection (SW)}

Stiffened welded connection is identical to the unstiffened joint, except that there is a stiffening plate, $10 \mathrm{~mm}$, welded between the two rectangular hollow sections, as shown in Figure (14.b).

\section{c) Welded internal sleeve (WS)}

It is a knee joint just like unstiffened welded connection, but with an internal hollow steel sleeve of thickness $10 \mathrm{~mm}$, as shown in Figure (14.c) and is welded inside the knee connections. Internal sleeve is composed of two hollow steel sections which cut at the same angle $105 \mathrm{o}$ and welded to each other with perfect contact in ABAQUS ${ }^{\circledR}$. Finally, the two RHS were welded together to the sleeve. Full details are available in Wilkinson and Hancock [29].
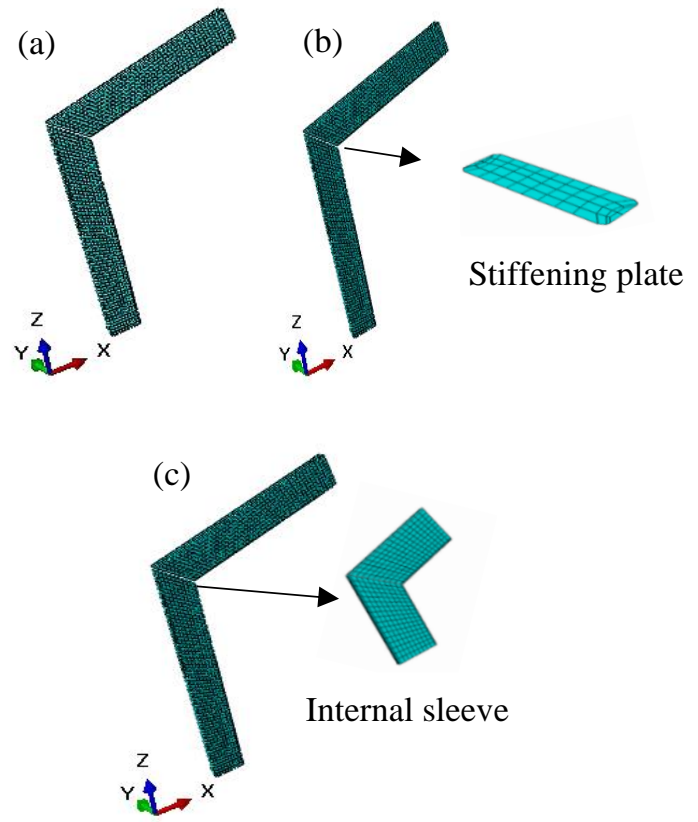

Figure 14: Unstiffened connection US (a), stiffened connection SW (b), and welded sleeve connection WS (c).

\subsubsection{Loads and Boundary Conditions}

Knee joint models were loaded using displacement control which was applied as a vertical displacement at the center top of RHS2 in Z direction. To simulate the test arrangement in [29] and its boundary conditions, displacement U2 was restrained in two places as shown in Figure (15), to prevent out of plane deformation. Displacement U1 and U3 were restrained at the base of RHS1 while for base of RHS2, only displacement U1 was restrained.

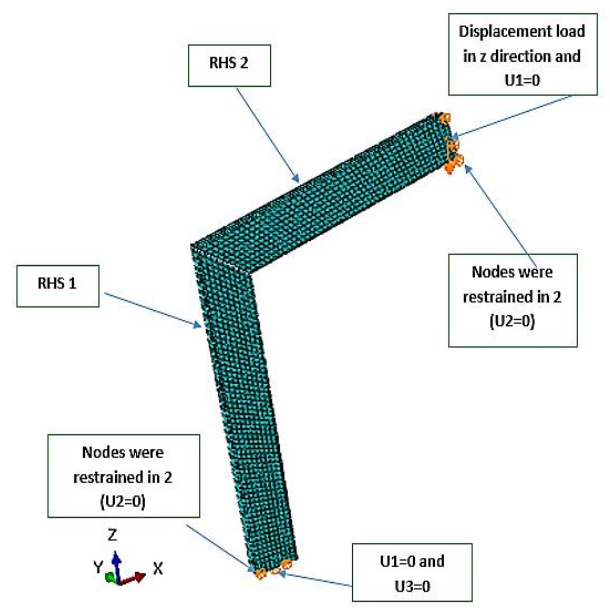

(a) 


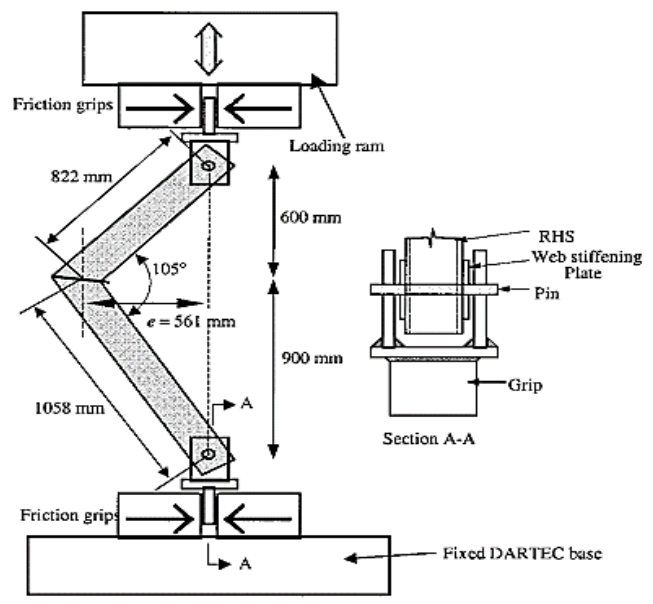

(b)

Figure 15: The three-dimensional finite element knee joint model (a), and test setup (b).

\subsubsection{Nonlinear Solution}

The Newton-Raphson method was used to solve three knee joints with maximum number of load increments $=100$, initial increment size $=0.01$, maximum increment size $=0.1$ and minimum increment size $=0.00001$.

\subsubsection{Material Modeling}

Wilkinson and Hancock [29] presented the stress-strain curves for two steel grades obtained from tensile test where stiffened and unstiffened knee joints are used C450 grade for specimens KJ01 and KJ05 in [29] while the other welded sleeve connection is used $\mathrm{C} 350$ grade for specimen $\mathrm{KJ} 13$ in [29]. Poisson's ratio was taken 0.3 for all analyses and modulus of elasticity for stiffened and unstiffened knee joints were taken $218000 \mathrm{MPa}$ and for welded sleeve connection was taken $210000 \mathrm{MPa}$. As mentioned before in section 2.1.2, the stress-strain curves were converted to true stress and logarithmic plastic strain as shown in Figure (16).

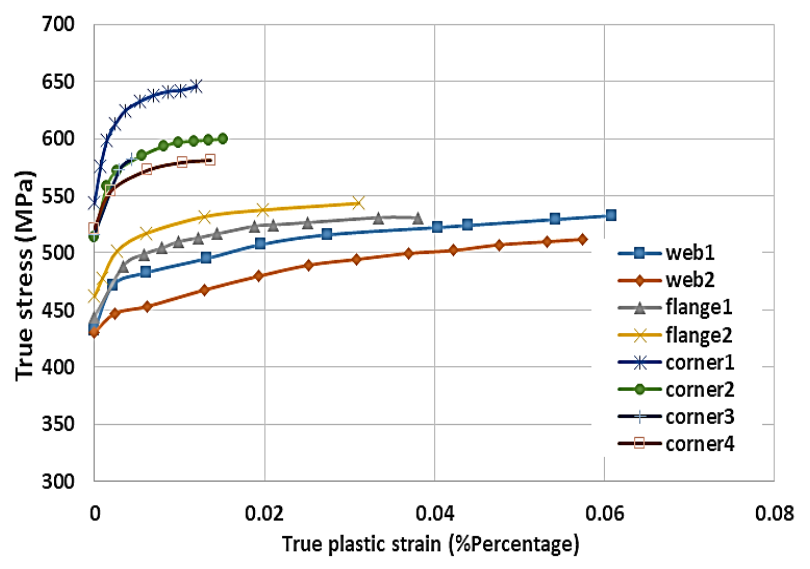

(a)

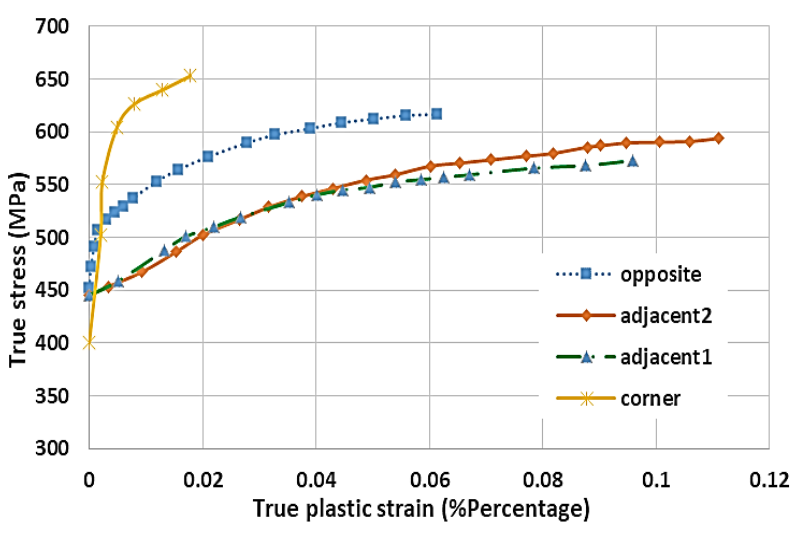

(b)

Figure 16: True Stress-strain curve for stiffened and unstiffened knee joints grade $\mathrm{C450}$ steel (a), welded sleeve connection grade $\mathbf{C 3 5 0}$ steel (b).

\subsubsection{Element Type and Mesh Density}

Quadratic thin shell elements in finite element analysis like S8R5 were used to verify results of three knee joints experimental tests presented in Wilkinson and Hancock [29]. A rigid quadrilateral R3D3 element was used for rigid plate in stiffened welded connection. R3D3 has four nodes with only three translational degrees of freedom per node. Medium mesh was used for all three knee joints to save time and to give appropriate results compared to tests as stated in section 2.1.7.1.

\subsubsection{Initial Imperfections}

Both linear and static non-linear analyses were used as explained before in section 2.1.5 to verify the results of the experimental tests of three knee joints. For all knee joints, the magnitude of imperfection was again chosen as a percent of thickness (0.1t, 0.5t, and 1.0t) [30, 34, 35].

\subsubsection{Residual Stresses}

Membrane and longitudinal bending residual stresses distributions were defined to all three knee joints as illustrated in section 2.1.6. It was achieved by defining local coordinates for each element then variations of residual stresses were modelled outside and inside through the thickness of the cold formed hollow sections.

\subsubsection{Results and Discussion}

Finite element analysis provided 108 models to predict the behavior of stiffened, unstiffened knee joints, and welded sleeve connections to resemble the experimental tests in Wilkinson and Hancock [29]. Each knee joint was analyzed using three values of initial imperfections $(0.1 \mathrm{t}, 0.5 \mathrm{t}$, and 1.0 t) and twelve buckling mode shapes. Typical load versus displacement curves for 24 models were illustrated in Figure (17) showing a comparison between the experimental tests [29] and the analytical results for the unstiffened (specimen KJ05 [29]) and welded sleeve connection (specimen KJ13 [29]) using twelve buckling mode shapes and with value of imperfection (0.1t). 


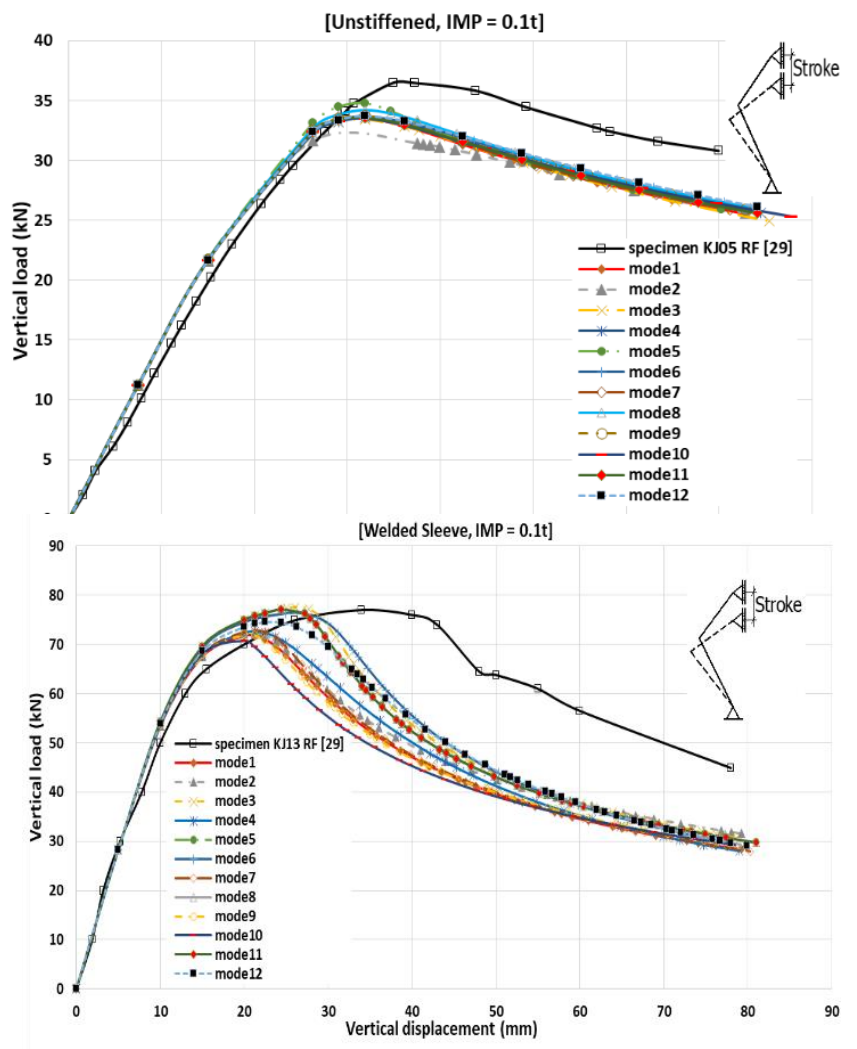

Figure 17: Comparison between two knee joints with value of imperfection (0.1t) using 12 buckling mode shapes.

All modes of failure were accurately checked for all three knee joints models. It can be noted that the initial imperfection of $(0.1 \mathrm{t})$ gives the best results in knee joints compared to test results in [29]. It resulted in that mode 8 is the most critical mode for unstiffened and mode 9 for stiffened knee joints, while for welded internal sleeve connection, mode 3 was the critical mode. Those modes show good agreement of failures which obtained from finite element analysis with those in the experimental tests in [29], as shown in Figure 18.
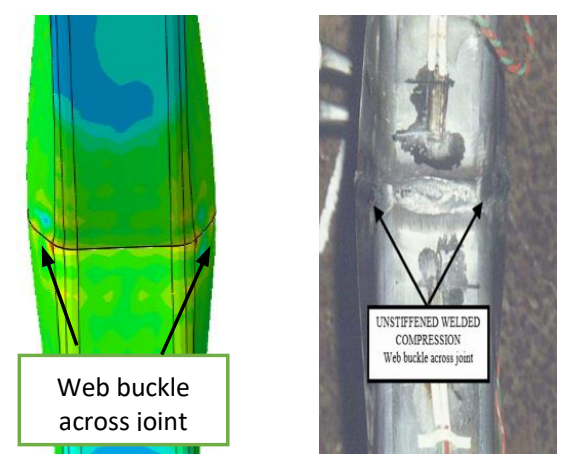

(a)

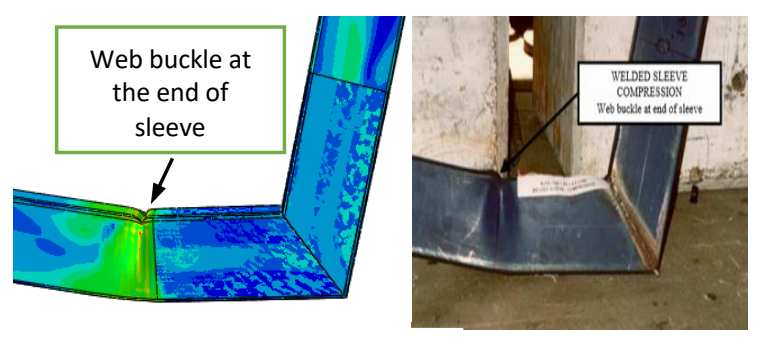

(b)

Figure 18: Comparison between test and FE simulation of specimen KJ05 (a) and KJ13 (b).

As shown in Figure 19, an analysis was carried out for models without imperfections (IMP) and residual stresses (RS) and other models with different values of imperfections $(0.1 \mathrm{t}, 0.5 \mathrm{t}$ and $1.0 \mathrm{t})$.

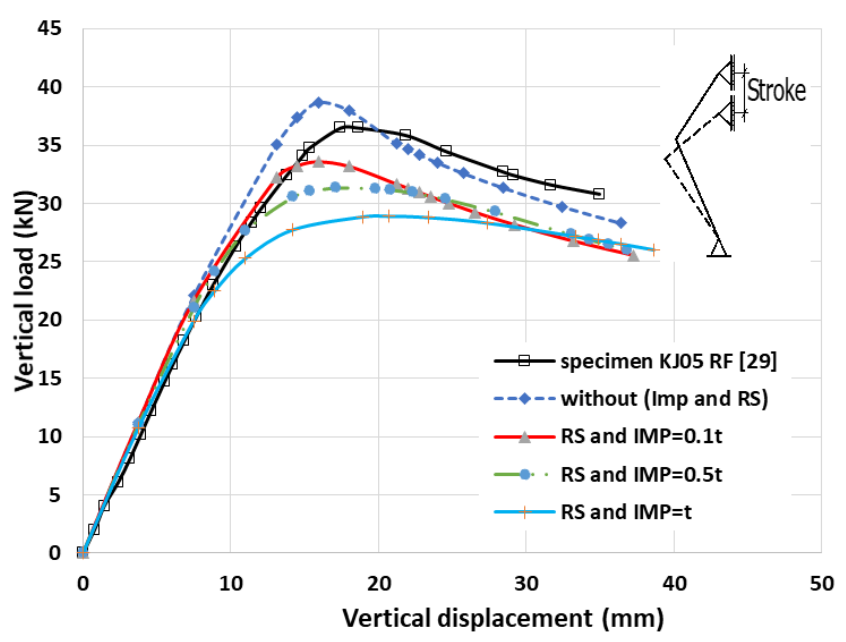

Figure 19: sensitivity to residual stresses and different imperfection for the most critical buckling mode (mode 8) for unstiffened knee joint.

For the most critical buckling mode for unstiffened knee joints and residual stresses in Key and Hancock [31], the analyses showed the results are sensitive to the presence of initial imperfections and residual stresses.

The analysis was able to capture the initial stiffness but not the ultimate load when both residual stresses and imperfections were present. On the other hand, neglecting them enabled the analysis to predict both initial stiffness and the ultimate load within an acceptable accuracy. Summary of verifications is shown in Table (1). 
Table 1: Summary of finite element analysis for frame and knee joint models.

\begin{tabular}{|l|c|c|c|c|l|}
\hline \multicolumn{1}{|c|}{ Specimen } & Cross-section & Knee Joint Type & Steel Grade & Solution Control & \multicolumn{1}{c|}{ Failure mode } \\
\hline Frame Test 3 [24] & RHS $200 \times 100 \times 4$ & $\begin{array}{c}\text { Beam directly } \\
\text { welded to column }\end{array}$ & C350 & Load Control & $\begin{array}{l}\text { Local buckling near the } \\
\text { base of both columns and } \\
\text { top of each column }\end{array}$ \\
\hline KJ01 [29] & RHS $150 \times 50 \times 4$ & SW & C450 & Displacement Control & Local buckle beside knee \\
\hline KJ05 [29] & RHS $150 \times 50 \times 4$ & US & C450 & Displacement Control & Local buckle inside of knee \\
\hline KJ13 [29] & RHS $150 \times 50 \times 4$ & WS & C350 & Displacement Control & $\begin{array}{l}\text { Local buckle at the end of } \\
\text { sleeve }\end{array}$ \\
\hline
\end{tabular}

\section{PARAMETRIC STUDY OF FRAME.}

Based on the verification done of the nonlinear finite element analysis carried out in the present study versus the experimental work from previous research, a parametric study was carried out. This study concentrates on investigation of the behavior of steel frame consisted of rectangular cold formed hollow section. The parametric study includes frames with great variability in geometrics, height, spans, and thicknesses. With wide range of results, it is then possible to obtain behavior curves for ultimate loads and conclude modes of failure in the studied models.

\subsection{Model Description}

A total of 144 finite element models of cold formed steel frames with major axis bending were carried out. The steel frames were divided into 16 groups, the groups from 1 to 8 (72 frames) were $200 \times 100 \mathrm{~mm}$ rectangular cold formed hollow sections and the other groups, from 9 to 16 (72 frames) were $150 \times 50 \mathrm{~mm}$ rectangular cold formed hollow sections. For each cross-section, different thicknesses were used $1 \mathrm{~mm}, 2 \mathrm{~mm}$, and $4 \mathrm{~mm}$. For each frame, analysis of three cases was carried out depending on the presence of initial geometric imperfection and residual stresses. In Case 1 , the frame was modelled without initial imperfections (Wo IMP) and residual stresses (Wo RS). While in Case 2, initial imperfections were not existing (Wo IMP), but residual stresses (W RS) were existing. Finally, in Case 3, frame was modelled using both initial imperfections (W IMP) and residual stresses (W RS). Every three groups consisted of constant span ( $\mathrm{L}=4 \mathrm{~m}, 6 \mathrm{~m}$, and $8 \mathrm{~m})$ with different heights $(\mathrm{H}=4 \mathrm{~m}, 6 \mathrm{~m}$, and $8 \mathrm{~m})$. Expect for frames with $4 \mathrm{~m}$ span, the frames were modelled with only $\mathrm{H}=4 \mathrm{~m}$ and $6 \mathrm{~m}$. The models' relevant parameters details are given in Table (2).

Table 2: Dimensions of frames and their cross-sections used in the parametric study.

\begin{tabular}{|c|c|c|c|}
\hline $\begin{array}{c}\text { Cross- } \\
\text { Section }\end{array}$ & $\begin{array}{c}\text { Thickness } \\
(\mathbf{m m})\end{array}$ & $\begin{array}{c}\text { Span } \\
(\mathbf{m})\end{array}$ & Height $(\mathbf{m})$ \\
\hline $\begin{array}{c}200 \mathrm{~mm} \times 100 \\
\mathrm{~mm}\end{array}$ & 1 & 4 & 4 \\
\cline { 1 - 1 } $\begin{array}{c}150 \mathrm{~mm} \times 50 \\
\mathrm{~mm}\end{array}$ & 2 & 6 & 6 \\
\hline
\end{tabular}

\subsection{Material Modelling}

All parts of the examined frames were modeled using C350 steel grade, summarized in Figure (3), as presented in [24].

\subsection{Element Type and Mesh Density}

Linear element type S4R5 and medium mesh were used for all models in the parametric study, based on the verification study in section 2.1.3.

\subsection{Nonlinear Solution, Loading Type and Boundary Conditions}

Static-riks method was chosen in the analysis of all parametric study models to detect post-peak behavior as previously discussed. All frames' models were loaded using load control method which was applied as equal vertical loads at the center top of each column accompanied with horizontal load at left column as shown in Figure (20). A 200 $\mathrm{kN}$ force was applied at the top of each column. The vertical to horizontal load ratio was chosen approximately 8:1 as per the test data in [24].

All the 144 frames had two hinged bases which were modelled by restraining $\mathrm{U} 1, \mathrm{U} 2$, and $\mathrm{U} 3$ displacements at the centroid of the two columns. Three places were restrained to prevent out of plane displacement of the frames for which U2 was set to 0 in three locations, as shown in Figure (20). 


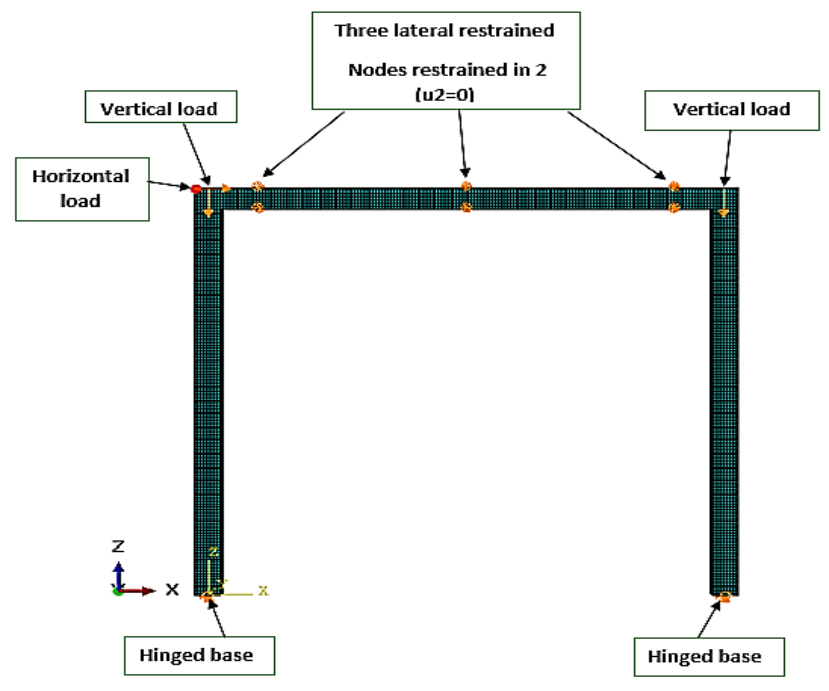

Figure 20: Finite element meshing, loading, and boundary conditions for frames used in the parametric study.

\subsection{Initial Geometric Imperfections and}

\section{Residual stresses}

Both initial imperfection and residual stresses were present in the nonlinear analysis for all the frames based on the verification results discussed previously in the present study. The initial imperfection was modelled using the buckling mode 2 and magnitude $0.5 \mathrm{t}$ while the residual stresses on the cold formed sections were modelled as discussed in the previous sections.

\subsection{Results and Discussion}

The parameters under investigation in this section are, the effect of the method of representing imperfection, the effect of cross-section thickness, the effect of frame span value, the effect of frame height, the effect of cross-section size.

\subsubsection{Effect of initial imperfection representing} method

Before conducting the parametric study, a study of the first group of the parametric study was carried out for a 108 finite element models to analyze each frame with case 3 only and using a 12 buckling mode shapes accompanied with three values of the initial imperfection, as listed in Table (3). The goal of doing such comparison is the show the sensitivity of the analysis results according to the value assigned to the initial imperfection and the selected mode shape. The increase or decrease in the value of the ultimate load $(\mathrm{Pu})$ for each method of imperfection is shown in Table (3) as a percentage related to the verified value of imperfection $(0.5 \mathrm{t})$ with mode shape $2(\mathrm{Pu}, 0.5 \mathrm{t}(2))$.

Table 3: Effect of imperfection value and mode shape on the ultimate load on the value of the ultimate load, Pu, for case 3 (with initial imperfections and residual stresses).

\begin{tabular}{|c|c|c|c|c|c|c|c|c|c|c|c|c|c|c|c|c|c|c|}
\hline \multirow{3}{*}{ 莺 } & \multirow{3}{*}{ 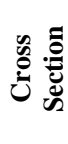 } & \multirow{3}{*}{$\underset{\mathbf{m m}}{\mathbf{t},}$} & \multirow{3}{*}{$\begin{array}{l}\mathbf{L}, \\
\mathbf{m}\end{array}$} & \multirow{3}{*}{$\begin{array}{l}\mathbf{H}, \\
\mathbf{m}\end{array}$} & \multirow{3}{*}{ 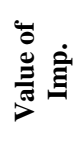 } & \multirow{3}{*}{ 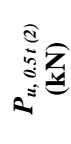 } & \multicolumn{12}{|c|}{$\%$ increase/decrease in $\boldsymbol{P}_{u}$ for different mode shapes w.r.t $\boldsymbol{P}_{u, 0.5 t(2)}$} \\
\hline & & & & & & & \multicolumn{12}{|c|}{ Imperfection Mode No. } \\
\hline & & & & & & & 1 & 2 & 3 & 4 & 5 & 6 & 7 & 8 & 9 & 10 & 11 & 12 \\
\hline \multirow{3}{*}{1} & \multirow{9}{*}{ 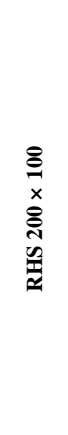 } & \multirow{3}{*}{1} & \multirow{9}{*}{4} & \multirow{9}{*}{4} & $0.1 t$ & \multirow{3}{*}{14.65} & -13.45 & -8.67 & 1.98 & 2.25 & 1.98 & -0.14 & 2.05 & -0.55 & 1.02 & -0.55 & -21.77 & -6.01 \\
\hline & & & & & $0.5 t$ & & -4.16 & 0.00 & -4.10 & 1.84 & -3.69 & -0.27 & -4.03 & -0.89 & -0.89 & -24.71 & 1.16 & 1.09 \\
\hline & & & & & $1.0 t$ & & -4.23 & -0.82 & -6.08 & 1.43 & -5.60 & -0.07 & -4.10 & -5.19 & -11.40 & -20.34 & 0.34 & -7.17 \\
\hline \multirow{3}{*}{2} & & \multirow{3}{*}{2} & & & $0.1 t$ & \multirow{3}{*}{43.66} & 2.13 & 10.74 & 2.27 & 2.22 & 2.18 & 14.41 & 2.18 & 14.50 & 2.38 & 0.30 & 2.36 & 2.36 \\
\hline & & & & & $0.5 \mathrm{t}$ & & -1.97 & 0.00 & -3.02 & -0.85 & -1.60 & -0.07 & -1.67 & -2.02 & -0.30 & -3.11 & 1.76 & 1.79 \\
\hline & & & & & $1.0 \mathrm{t}$ & & -3.41 & -1.90 & -4.51 & -0.02 & -3.14 & -2.47 & -3.34 & -4.21 & -1.86 & -5.79 & 1.76 & 0.62 \\
\hline \multirow{3}{*}{3} & & \multirow{3}{*}{4} & & & $0.1 t$ & \multirow{3}{*}{122.58} & -0.16 & 0.00 & 0.10 & 0.10 & -1.35 & 0.29 & -2.67 & 0.30 & -2.70 & 0.07 & 0.78 & -0.50 \\
\hline & & & & & $0.5 t$ & & -0.44 & 0.00 & 0.00 & -2.22 & -4.57 & -1.66 & -4.85 & -0.97 & -4.24 & -1.44 & -5.26 & -2.55 \\
\hline & & & & & $1.0 \mathrm{t}$ & & -0.86 & 0.00 & 0.00 & -5.45 & -7.43 & -4.86 & -7.00 & -4.14 & -7.30 & -3.41 & -8.16 & -4.50 \\
\hline
\end{tabular}

The results in Table (3) show that the value of the ultimate load can be greatly affected by the value of initial imperfection and the selected mode shape. That is in some cases, a decrease in the value of ultimate load is up to $24.7 \%$. Moreover, the average of all the absolute values is about $3.46 \%$ which is a significant ratio.

\subsubsection{Effect of initial imperfection and residual} stresses

Tables 4 and 5 illustrate the results for 48 models with constant value of initial imperfections equal to (0.5t) of RHS with buckling mode shape 2. Every frame was analyzed using 3 cases under the same loading and boundary conditions. In Case 1, frames were modelled without imperfection and residual stresses. While in Case 2, frames were modelled without imperfection, but with residual stresses. Finally, is Case 3, frames both imperfection and residual stresses existed in the model. The ultimate loads for each case were shown in Tables 4 and 5. In addition, the percentage of decrease in these ultimate loads due to the presence of residual stresses and/or initial imperfections is 
shown referenced to the ultimate load for frames without residual stresses and initial imperfections.

Tahle 4: FF.A results for 72 models with RHS $200 \times 100$ with/withont imnerfection and residual stresses.

\begin{tabular}{|c|c|c|c|c|c|c|c|c|c|c|c|}
\hline 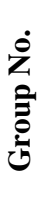 & 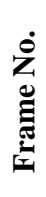 & 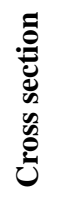 & $\begin{array}{c}\mathrm{t} \\
(\mathbf{m m})\end{array}$ & $\begin{array}{c}\mathbf{L} \\
(\mathbf{m})\end{array}$ & $\begin{array}{c}\mathbf{H} \\
(\mathbf{m})\end{array}$ & $\begin{array}{c}P_{u I} \\
(\mathbf{k N})\end{array}$ & $\begin{array}{c}P_{u 2} \\
(\mathbf{k N})\end{array}$ & 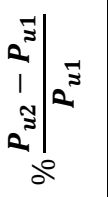 & $\begin{array}{c}P_{u 3} \\
(\mathbf{k N})\end{array}$ & 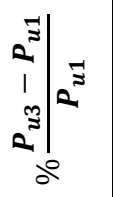 & Type of failure \\
\hline \multirow{3}{*}{1} & 1 & \multirow{24}{*}{ 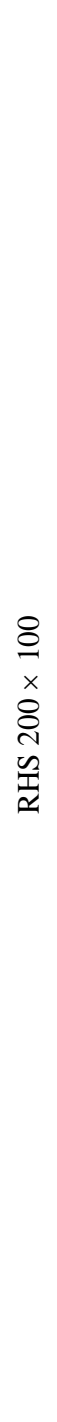 } & 1 & \multirow{6}{*}{4} & \multirow{3}{*}{4} & 14.936 & 14.804 & -0.884 & 14.587 & -2.337 & RCcLB (PH), LCLB, LbLB (PH) \\
\hline & 2 & & 2 & & & 46.452 & 44.668 & -3.841 & 43.658 & -6.015 & RCcLB (PH) \\
\hline & 3 & & 4 & & & 124.659 & 122.633 & -1.625 & 122.578 & -1.669 & RCcLB (PH), LbLB (PH) \\
\hline \multirow{3}{*}{2} & 4 & & 1 & & \multirow{3}{*}{6} & 9.739 & 9.078 & -6.787 & 7.822 & -19.684 & RCLB \\
\hline & 5 & & 2 & & & 28.793 & 27.248 & -5.366 & 25.756 & -10.548 & RCcLB (PH) \\
\hline & 6 & & 4 & & & 73.251 & 70.269 & -4.071 & 70.268 & -4.072 & RCcLB (PH), LbLB (PH) \\
\hline \multirow{3}{*}{3} & 7 & & 1 & \multirow{9}{*}{6} & \multirow{3}{*}{4} & 14.386 & 14.038 & -2.419 & 13.813 & -3.983 & RCcLB (PH), LbLB (PH) \\
\hline & 8 & & 2 & & & 44.262 & 42.084 & -4.921 & 41.195 & -6.929 & RCcLB (PH) \\
\hline & 9 & & 4 & & & 116.620 & 113.724 & -2.483 & 113.702 & -2.502 & RCcLB (PH), LbLB (PH) \\
\hline \multirow{3}{*}{4} & 10 & & 1 & & \multirow{3}{*}{6} & 9.352 & 8.647 & -7.538 & 8.748 & -6.459 & RCcLB (PH), LbLB, LCLB, RCLB \\
\hline & 11 & & 2 & & & 27.390 & 25.414 & -7.214 & 24.658 & -9.974 & $\mathrm{RCcLB}(\mathrm{PH})$ \\
\hline & 12 & & 4 & & & 69.150 & 66.020 & -4.526 & 66.020 & -4.526 & RCcLB (PH), LbLB (PH) \\
\hline \multirow{3}{*}{5} & 13 & & 1 & & \multirow{3}{*}{8} & 6.447 & 6.255 & -2.978 & 5.562 & -13.727 & RCLB \\
\hline & 14 & & 2 & & & 18.978 & 17.784 & -6.290 & 16.912 & -10.889 & RCcLB (PH) \\
\hline & 15 & & 4 & & & 46.450 & 43.789 & -5.729 & 43.789 & -5.729 & RCcLB (PH) \\
\hline \multirow{3}{*}{6} & 16 & & 1 & \multirow{9}{*}{8} & \multirow{3}{*}{4} & 13.837 & 13.443 & -2.849 & 13.475 & -2.616 & $\operatorname{LbLB}(\mathrm{PH}), \mathrm{RCcLB}(\mathrm{PH})$ \\
\hline & 17 & & 2 & & & 42.255 & 39.978 & -5.391 & 39.680 & -6.094 & RCcLB (PH) \\
\hline & 18 & & 4 & & & 109.994 & 106.355 & -3.309 & 106.354 & -3.310 & LbLB (PH), RCcLB (PH) \\
\hline \multirow{3}{*}{7} & 19 & & 1 & & \multirow{3}{*}{6} & 9.010 & 8.509 & -5.566 & 8.425 & -6.494 & RCcLB (PH), LbLB, LCLB, RCLB \\
\hline & 20 & & 2 & & & 26.114 & 24.525 & -6.087 & 23.199 & -11.163 & RCcLB (PH) \\
\hline & 21 & & 4 & & & 65.586 & 62.305 & -5.003 & 62.305 & -5.003 & RCcLB (PH) \\
\hline \multirow{3}{*}{8} & 22 & & 1 & & \multirow{3}{*}{8} & 6.241 & 6.033 & -3.338 & 5.206 & -16.590 & $\mathrm{RCLB}(\mathrm{PH})$ \\
\hline & 23 & & 2 & & & 18.229 & 17.085 & -6.274 & 17.085 & -6.274 & RCcLB (PH) \\
\hline & 24 & & 4 & & & 44.319 & 41.681 & -5.952 & 41.681 & -5.952 & RCcLB (PH), LbLB (PH) \\
\hline
\end{tabular}

RHS: Rectangular hollow section.

LbLB: Left beam local buckling.

LCLB: Left column local buckling

RCFHS: Rectangular cold formed hollow section

RCLB: Right column local buckling.

RCcLB (PH): Right column connection local buckling due to plastic hinge formation. 
Table 5: FEA results for 72 models with RHS 150×50 with/without imperfection and residual stresses.

\begin{tabular}{|c|c|c|c|c|c|c|c|c|c|c|c|}
\hline $\begin{array}{l}\dot{Z} \\
\dot{Z} \\
\dot{0} \\
\dot{0}\end{array}$ & 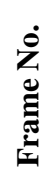 & 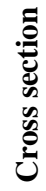 & $\underset{(\mathbf{m m})}{\mathbf{t}}$ & $\begin{array}{c}\mathbf{L} \\
(\mathbf{m})\end{array}$ & $\begin{array}{c}\mathbf{H} \\
(\mathbf{m})\end{array}$ & $\begin{array}{c}P_{u 1} \\
(\mathbf{k N})\end{array}$ & $\begin{array}{c}P_{u 2} \\
(\mathbf{k N})\end{array}$ & 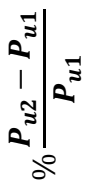 & $\begin{array}{c}P_{u 3} \\
(\mathbf{k N})\end{array}$ & 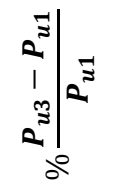 & Type of failure \\
\hline \multirow{3}{*}{9} & 25 & \multirow{24}{*}{ 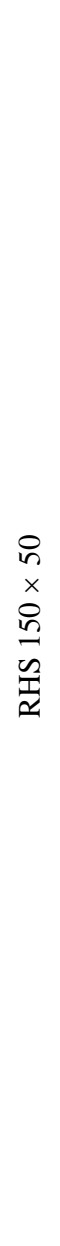 } & 1 & \multirow{6}{*}{4} & \multirow{3}{*}{4} & 8.375 & 7.967 & -4.880 & 7.757 & -7.379 & $\mathrm{RCcLB}(\mathrm{PH})$ \\
\hline & 26 & & 2 & & & 23.665 & 22.062 & -6.773 & 22.076 & -6.715 & $\mathrm{RCcLB}(\mathrm{PH})$ \\
\hline & 27 & & 4 & & & 52.018 & 49.694 & -4.469 & 49.692 & -4.471 & $\mathrm{RCcLB}(\mathrm{PH}), \operatorname{LbcLB}(\mathrm{PH})$ \\
\hline \multirow{3}{*}{10} & 28 & & 1 & & \multirow{3}{*}{6} & 5.258 & 4.844 & -7.875 & 4.614 & -12.249 & $\mathrm{RCcLB}(\mathrm{PH})$ \\
\hline & 29 & & 2 & & & 13.807 & 12.860 & -6.861 & 12.862 & -6.844 & $\mathrm{RCcLB}(\mathrm{PH})$ \\
\hline & 30 & & 4 & & & 29.364 & 27.489 & -6.387 & 27.533 & -6.235 & $\mathrm{RCcLB}(\mathrm{PH}), \mathrm{LbLB}(\mathrm{PH})$ \\
\hline \multirow{3}{*}{11} & 31 & & 1 & \multirow{9}{*}{6} & \multirow{3}{*}{4} & 7.895 & 7.492 & -5.097 & 7.301 & -7.526 & $\mathrm{RCcLB}(\mathrm{PH})$ \\
\hline & 32 & & 2 & & & 22.088 & 20.532 & -7.045 & 20.569 & -6.876 & $\mathrm{RCcLB}(\mathrm{PH})$ \\
\hline & 33 & & 4 & & & 48.373 & 45.610 & -5.713 & 45.609 & -5.715 & $\mathrm{RCcLB}(\mathrm{PH}), \operatorname{LbLB}(\mathrm{PH})$ \\
\hline \multirow{3}{*}{12} & 34 & & 1 & & \multirow{3}{*}{6} & 4.855 & 4.718 & -2.818 & 4.395 & -9.481 & $\mathrm{RCcLB}(\mathrm{PH})$ \\
\hline & 35 & & 2 & & & 12.987 & 12.079 & -6.990 & 12.081 & -6.980 & $\mathrm{RCcLB}(\mathrm{PH})$ \\
\hline & 36 & & 4 & & & 27.566 & 25.762 & -6.543 & 25.735 & -6.644 & $\mathrm{RCcLB}(\mathrm{PH}), \operatorname{LbLB}(\mathrm{PH})$ \\
\hline \multirow{3}{*}{13} & 37 & & 1 & & \multirow{3}{*}{8} & 3.346 & 3.172 & -5.218 & 3.172 & -5.218 & $\mathrm{RCcLB}(\mathrm{PH})$ \\
\hline & 38 & & 2 & & & 8.665 & 8.062 & -6.954 & 8.062 & -6.955 & $\mathrm{RCcLB}(\mathrm{PH})$ \\
\hline & 39 & & 4 & & & 18.111 & 16.766 & -7.428 & 16.761 & -7.456 & $\mathrm{RCcLB}(\mathrm{PH})$ \\
\hline \multirow{3}{*}{14} & 40 & & 1 & \multirow{9}{*}{8} & \multirow{3}{*}{4} & 7.552 & 7.096 & -6.046 & 7.220 & -4.399 & $\mathrm{RCcLB}(\mathrm{PH})$ \\
\hline & 41 & & 2 & & & 20.738 & 19.317 & -6.854 & 18.250 & -12.000 & $\mathrm{RCcLB}(\mathrm{PH})$ \\
\hline & 42 & & 4 & & & 44.857 & 42.344 & -5.602 & 42.343 & -5.603 & $\mathrm{RCcLB}(\mathrm{PH}), \operatorname{LbLB}(\mathrm{PH})$ \\
\hline \multirow{3}{*}{15} & 43 & & 1 & & \multirow{3}{*}{6} & 4.637 & 4.391 & -5.288 & 4.201 & -9.405 & $\mathrm{RCcLB}(\mathrm{PH})$ \\
\hline & 44 & & 2 & & & 12.273 & 11.425 & -6.913 & 11.424 & -6.920 & $\mathrm{RCcLB}(\mathrm{PH})$ \\
\hline & 45 & & 4 & & & 26.092 & 24.203 & -7.237 & 24.232 & -7.126 & $\mathrm{RCcLB}(\mathrm{PH})$ \\
\hline \multirow{3}{*}{16} & 46 & & 1 & & \multirow{3}{*}{8} & 3.205 & 3.042 & -5.065 & 3.042 & -5.065 & $\mathrm{RCcLB}(\mathrm{PH})$ \\
\hline & 47 & & 2 & & & 8.239 & 7.683 & -6.753 & 7.683 & -6.753 & $\mathrm{RCcLB}(\mathrm{PH})$ \\
\hline & 48 & & 4 & & & 17.253 & 15.940 & -7.606 & 15.943 & -7.592 & $\mathrm{RCcLB}(\mathrm{PH})$ \\
\hline
\end{tabular}

LbLB (PH): Left beam local buckling due to plastic hinge formation.

LbcLB (PH): Left beam connection local buckling due to forming plastic hinge.

The results show the importance of accounting of both the initial imperfection and residual stresses as the percentage of decrease in the ultimate load can reach $19.68 \%$ for RHS $200 \times 100 \times 1 \mathrm{~mm}$ and $12.25 \%$ for RHS $150 \times 50 \times 1 \mathrm{~mm}$. Further, the average of the percentage of decrease in ultimate load is about $5.4 \%$ in case of considering residual stresses only while this average is about $7.2 \%$ in case of considering both imperfections and residual stresses.

\subsubsection{Effect of cross-section size}

It is obvious that for the frames having the same dimensions and cross-sectional dimensions with different thicknesses, the cross-section thickness ratio between different frames could represent the weight ratio between such frames. Therefore, the ratio $(\mathrm{Pu} / \mathrm{t})$ could be a good indicator of the ultimate load to weight ratio. Furthermore, for the sake of comparison between the efficiency of frames with different cross-section thicknesses it is a good practice to relate this ratio to that of the frame with cross-section thickness $1 \mathrm{~mm}$ as follows in equation 3 .

$$
\% \frac{P_{u}}{w}=\left(\frac{\frac{P_{u, t}}{t}}{\frac{P_{u, t=1 \mathrm{~mm}}}{1}}\right) \times 100
$$

Table 6 shows that the ultimate load to weight ratio could reach $165 \%$ frames with RHS $200 \times 100$ and thickness $2 \mathrm{~mm}$ and $225 \%$ for frames with RHS $200 \times 100$ and thickness 4 
$\mathrm{mm}$. On the other hand, for the frames with RHS $150 \times 50$ and thickness 2 and $4 \mathrm{~mm}$, the ratio could be up to $142 \%$, $160 \%$, respectively. The increase of cross-sectional thickness has great effect on the frame loading capacity because it changes section class from slender to noncompact and compact classes. This leads to elimination of local buckling effect and lets the effect of nonlinear global buckling and yielding in form of plastic hinge formation appear. Therefore, the improve of ultimate load to weight could increase by at least $126 \%$ as shown in Table 6 .
The elastic global buckling of such frames is not affected by the cross-section thickness, because all RHS sections of the same dimensions and different thicknesses almost have the same radius of gyration, i.e., the same column slenderness ratios. This leads to same allowable buckling stress of all sections.

Moreover, Table 6 shows that the reduction of the other section dimensions, $h$ and $b$, reduces the ultimate load capacity of the section. This is because the reduction of the cross-section area and modulus. In addition, the reduction of the cross-section leads to increase in the effect of global buckling as well as yielding of the cross section.

Table 6: Effect of cross-section thickness, $t$, on the ultimate load, $P_{u}$ for all 48 frame models with both imperfection and residual stresses (Case 3).

\begin{tabular}{|c|c|c|c|c|c|c|c|c|c|c|}
\hline \multirow{2}{*}{$\begin{array}{c}\mathbf{t} \\
(\mathbf{m m})\end{array}$} & \multirow{2}{*}{$\begin{array}{c}\mathbf{L} \\
(\mathbf{m})\end{array}$} & \multirow{2}{*}{$\begin{array}{c}\mathbf{H} \\
(\mathbf{m})\end{array}$} & \multirow{2}{*}{$\underset{\mathbb{E}}{\stackrel{\Xi}{*}}$} & \multicolumn{3}{|c|}{ RHS $200 \times 100$} & \multirow{2}{*}{ 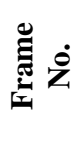 } & \multicolumn{3}{|c|}{ RHS $150 \times 50$} \\
\hline & & & & $\begin{array}{c}\boldsymbol{P}_{u} \\
(\mathbf{k N})\end{array}$ & $\begin{array}{c}P_{u} / t \\
(\mathbf{k N} / \mathbf{m m})\end{array}$ & $\% P_{u} / w$ & & $\begin{array}{c}\boldsymbol{P}_{u} \\
(\mathbf{k N})\end{array}$ & $\begin{array}{c}P_{u} / t \\
(\mathbf{k N} / \mathbf{m m})\end{array}$ & $\% P_{u} / w$ \\
\hline 1 & \multirow{6}{*}{4} & \multirow{3}{*}{4} & 1 & 14.587 & 14.587 & 100 & 25 & 7.757 & 7.757 & 100 \\
\hline 2 & & & 2 & 43.658 & 21.829 & 150 & 26 & 22.076 & 11.038 & 142 \\
\hline 4 & & & 3 & 122.578 & 30.645 & 210 & 27 & 49.692 & 12.423 & 160 \\
\hline 1 & & \multirow{3}{*}{6} & 4 & 7.822 & 7.822 & 100 & 28 & 4.614 & 4.614 & 100 \\
\hline 2 & & & 5 & 25.756 & 12.878 & 165 & 29 & 12.862 & 6.431 & 139 \\
\hline 4 & & & 6 & 70.268 & 17.567 & 225 & 30 & 27.533 & 6.88325 & 149 \\
\hline 1 & \multirow{9}{*}{6} & \multirow{3}{*}{4} & 7 & 13.813 & 13.813 & 100 & 31 & 7.301 & 7.301 & 100 \\
\hline 2 & & & 8 & 41.195 & 20.598 & 149 & 32 & 20.569 & 10.2845 & 141 \\
\hline 4 & & & 9 & 113.702 & 28.426 & 206 & 33 & 45.609 & 11.40225 & 156 \\
\hline 1 & & \multirow{3}{*}{6} & 10 & 8.748 & 8.748 & 100 & 34 & 4.395 & 4.395 & 100 \\
\hline 2 & & & 11 & 24.658 & 12.329 & 141 & 35 & 12.081 & 6.0405 & 137 \\
\hline 4 & & & 12 & 66.02 & 16.505 & 189 & 36 & 25.735 & 6.43375 & 146 \\
\hline 1 & & \multirow{3}{*}{8} & 13 & 5.562 & 5.562 & 100 & 37 & 3.172 & 3.172 & 100 \\
\hline 2 & & & 14 & 16.912 & 8.456 & 152 & 38 & 8.062 & 4.031 & 127 \\
\hline 4 & & & 15 & 43.789 & 10.947 & 197 & 39 & 16.761 & 4.19025 & 132 \\
\hline 1 & \multirow{9}{*}{8} & \multirow{3}{*}{4} & 16 & 13.475 & 13.475 & 100 & 40 & 7.22 & 7.22 & 100 \\
\hline 2 & & & 17 & 39.68 & 19.840 & 147 & 41 & 18.25 & 9.125 & 126 \\
\hline 4 & & & 18 & 106.354 & 26.589 & 197 & 42 & 42.343 & 10.58575 & 147 \\
\hline 1 & & \multirow{3}{*}{6} & 19 & 8.425 & 8.425 & 100 & 43 & 4.201 & 4.201 & 100 \\
\hline 2 & & & 20 & 23.199 & 11.600 & 138 & 44 & 11.424 & 5.712 & 136 \\
\hline 4 & & & 21 & 62.305 & 15.576 & 185 & 45 & 24.232 & 6.058 & 144 \\
\hline 1 & & \multirow{3}{*}{8} & 22 & 5.206 & 5.206 & 100 & 46 & 3.042 & 3.042 & 100 \\
\hline 2 & & & 23 & 17.085 & 8.543 & 164 & 47 & 7.683 & 3.8415 & 126 \\
\hline 4 & & & 24 & 41.681 & 10.420 & 200 & 48 & 15.943 & 3.98575 & 131 \\
\hline
\end{tabular}

To show the effect of cross-section thickness as well as imperfection and residual stresses on ultimate load capacity, horizontal displacement, and mid-span vertical deflection in Figure 21, nine models were analyzed. All frames are belonged to the first group at which $\mathrm{L}=4 \mathrm{~m}$ and $\mathrm{H}=4 \mathrm{~m}$ model without imperfection and residual stresses

and model with residual stresses, but without imperfection and model with both imperfection and residual stresses. The 
first, three models were with cross-section thickness $\mathrm{t}=1$ $\mathrm{mm}$, the other three models were with thickness $2 \mathrm{~mm}$, and the last three models were with thickness $4 \mathrm{~mm}$. The steel frames with different cross- section thicknesses have different modes of failure.
Figure 21 illustrates the relationship between both vertical load versus horizontal displacement and vertical load versus vertical displacement at center of beam. As could be expected, all the plots show that increasing the thickness could greatly increases the ultimate load.
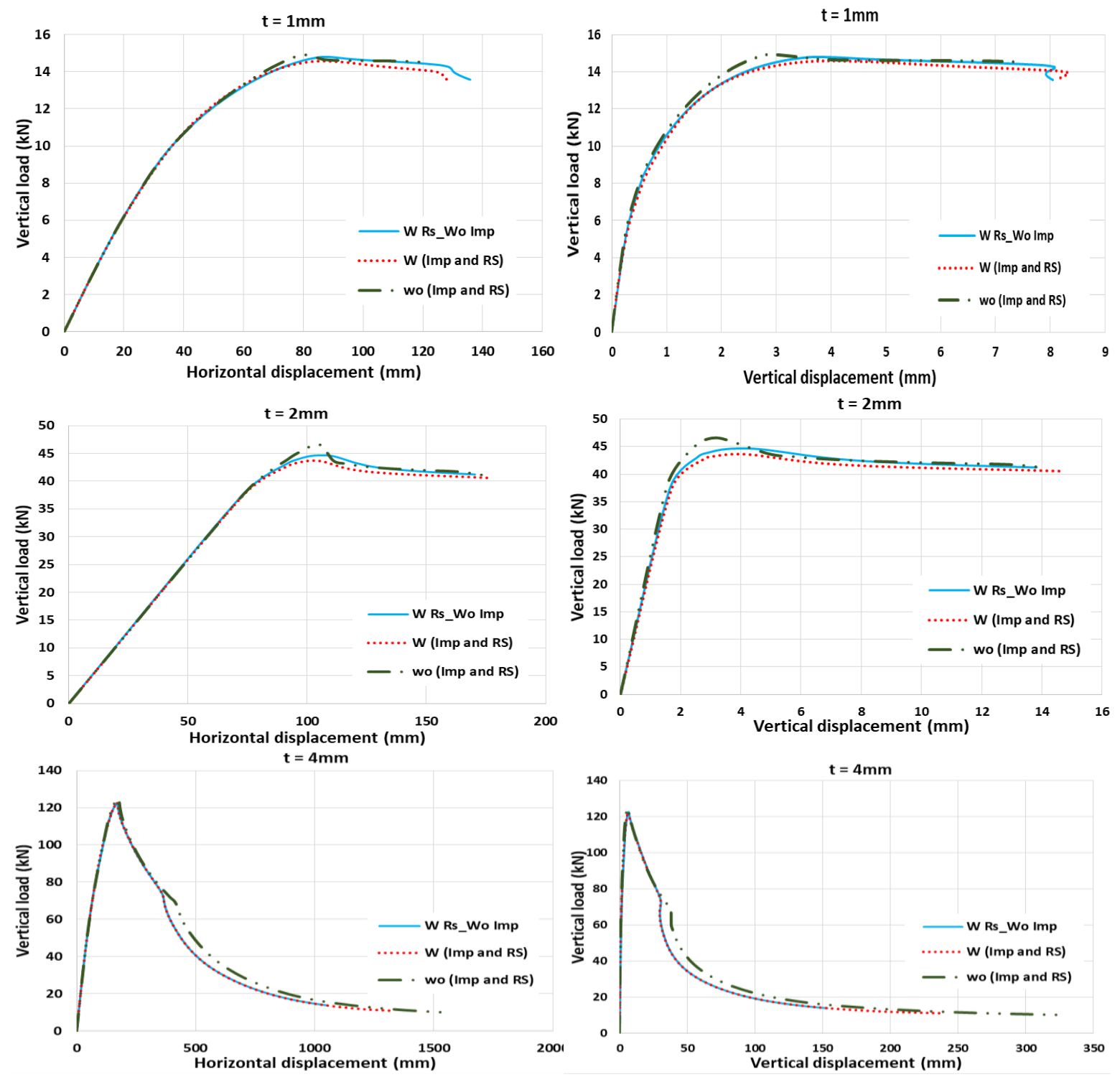

Figure 21: Load-displacement relation of the first group in the parametric study

Figure 22 shows summary of the ultimate load-thickness ratio, ultimate load-weight ratio, horizontal displacement, and vertical mid-span deflection at ultimate load for the only three of the above mentioned nine frames. These three frames incorporate the initial imperfection and residual stresses. Figure 22-a and -b emphasize on the effect of the cross-section thickness on the efficiency of the crosssection with respect to ultimate load capacity as discussed formerly in this section. While, Figure 22-c, and -d show that the value of horizontal and vertical displacement capacities at the value of ultimate load is smaller in vertical displacement than horizontal displacement with increasing the cross-section thickness. 


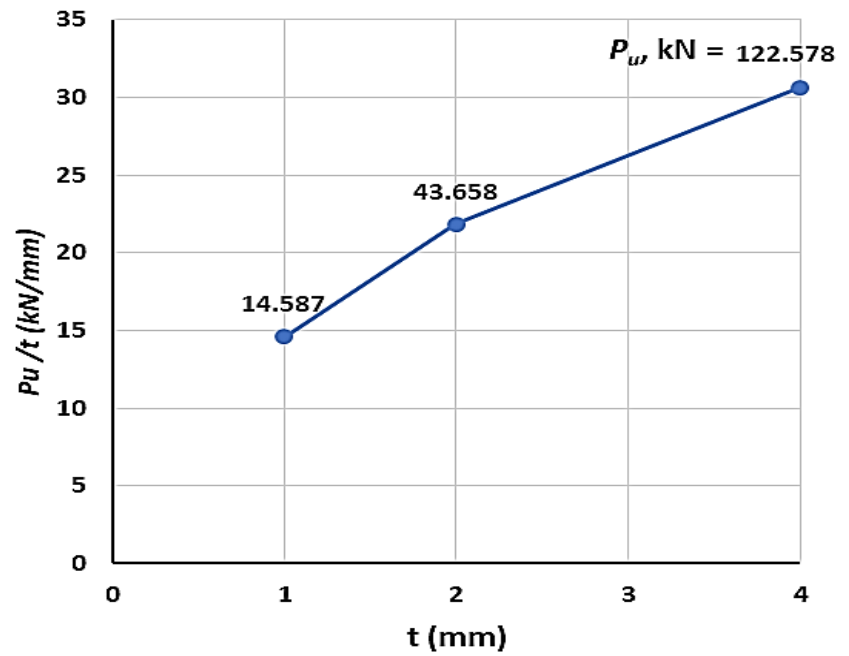

(a)

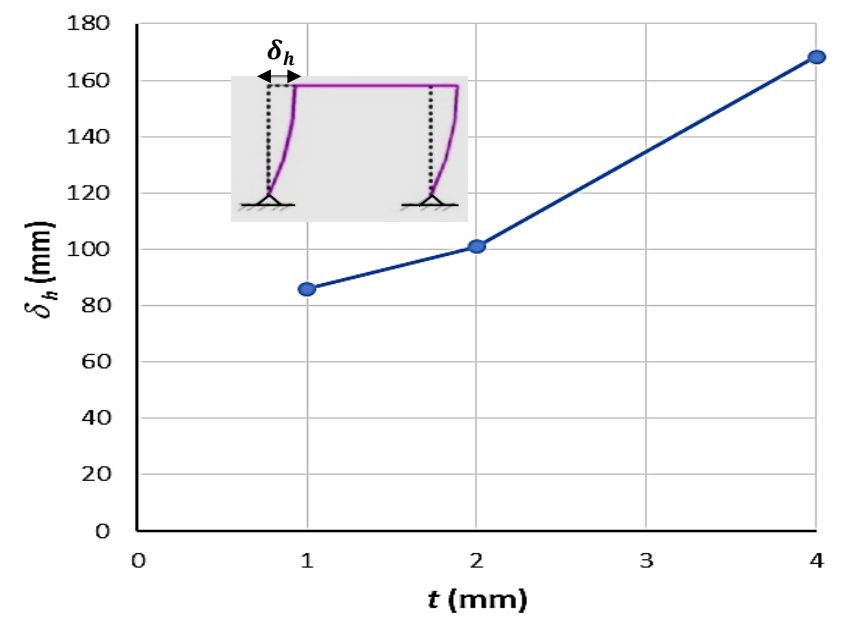

(c)

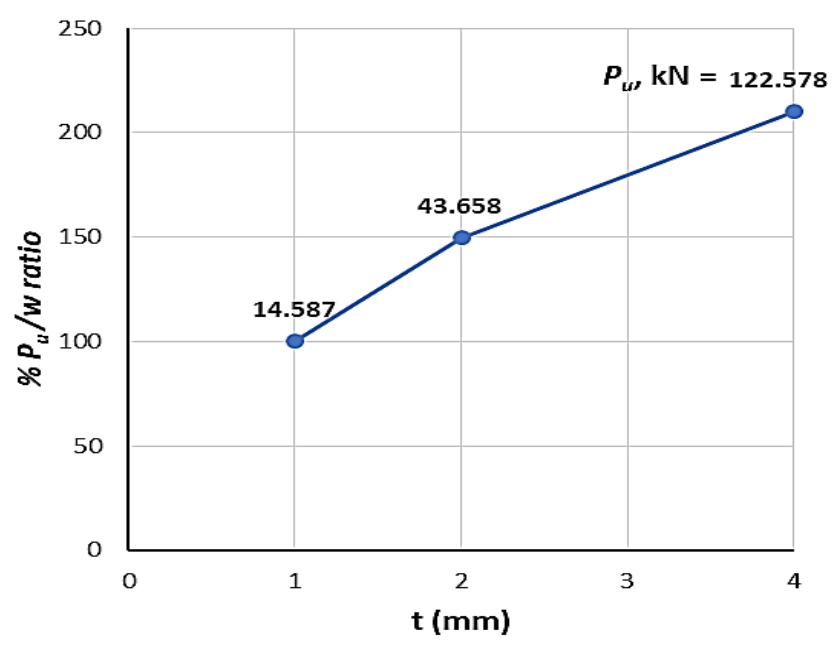

(b)

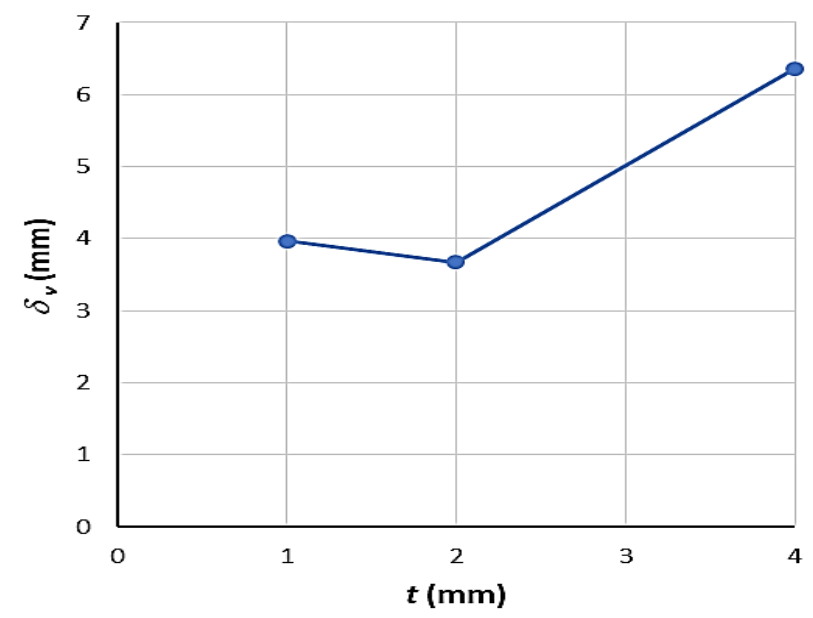

(d)

Figure 22: Effect of cross-section thickness for RHS 200×100 mm: a) displacement at ultimate -thickness, b) percentage ultimate load-weight ratio, c) horizontal displacement, and d) vertical mid-span deflection.

Figure 23 shows displacement at ultimate load for $(\mathrm{t}=$ $1 \mathrm{~mm})=14.587 \mathrm{kN}(\mathrm{a})$ and ultimate load-displacement ratio (b). Figures 23.a and 23.b illustrate the behavior of frame at ultimate load and emphasize that the value of ultimate load at horizontal and vertical displacement capacities is smaller in vertical displacement than horizontal displacement at increasing the cross-section thickness.

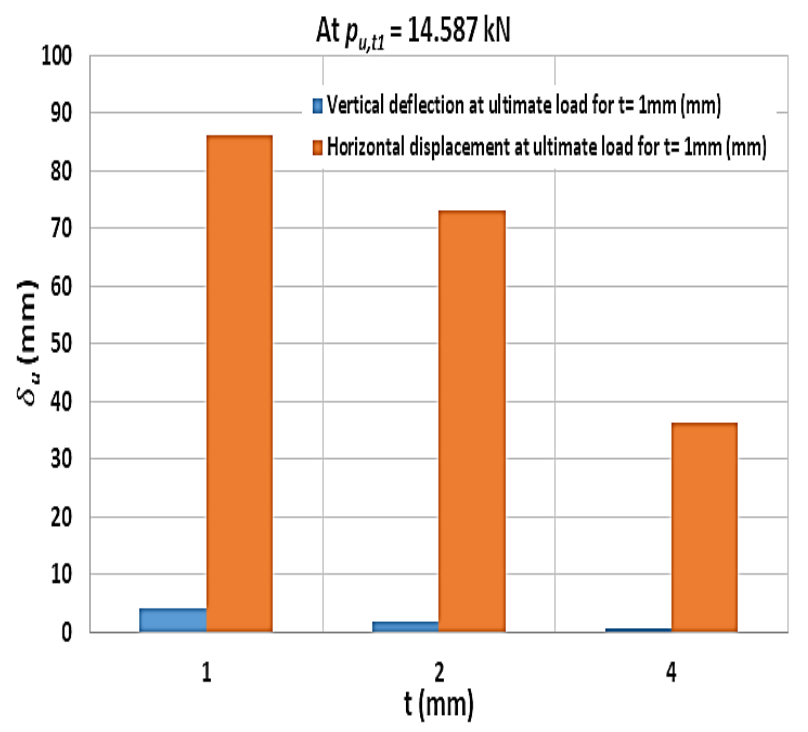




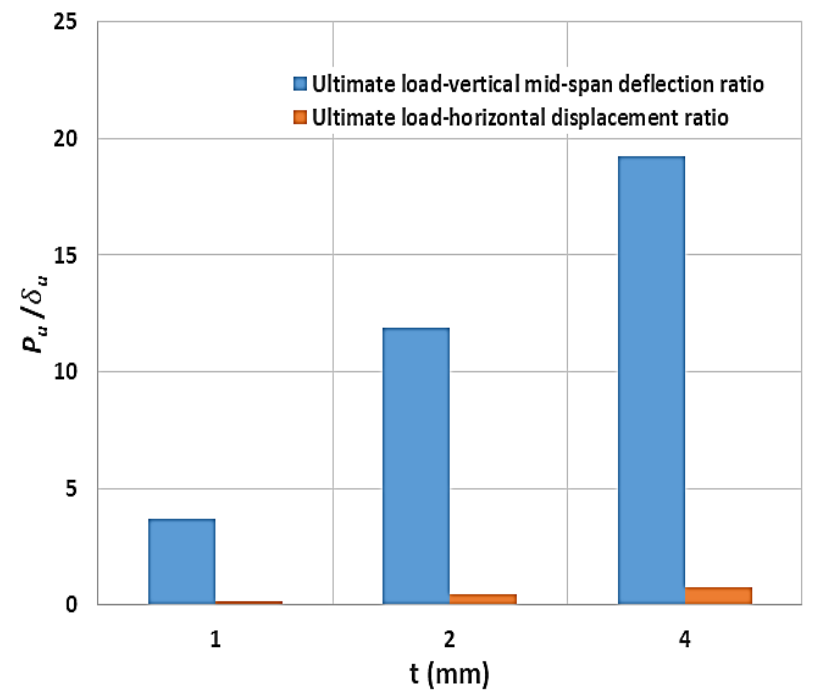

(b)

\subsubsection{Effect of frame dimensions}

Figure 24 shows a comparison between frames with spans $(4,6$ and $8 \mathrm{~m})$ and different heights $(4,6$, and $8 \mathrm{~m})$ versus maximum loads using case 3 of analysis. Figure 24 $-a,-b$ and $-c$ show the results of the groups of frames with cross section RHS $200 \times 100 \mathrm{~mm}$ while Figure $24-\mathrm{d}$, -e and -f is for frames with RHS $150 \times 50$. It is realized that the increase in frame height significantly reduces the ultimate load. Also, increasing the span length slightly reduces the ultimate load. This is because increasing the frame span length slightly increases the effective buckling length of the column, thus reduces the ultimate load capacity. On the other hand, increasing the height of the frame has greater effect on the column buckling length thus it reduces the ultimate load capacity by a much greater value than the effect of span length.

Figure 23: Effect of cross-section thickness for RHS 200×100 mm: a) displacement-thickness relation at ultimate load for $(t$ $=1 \mathrm{~mm})=14.587 \mathrm{kN}$, b) ultimate load-displacement ratio.

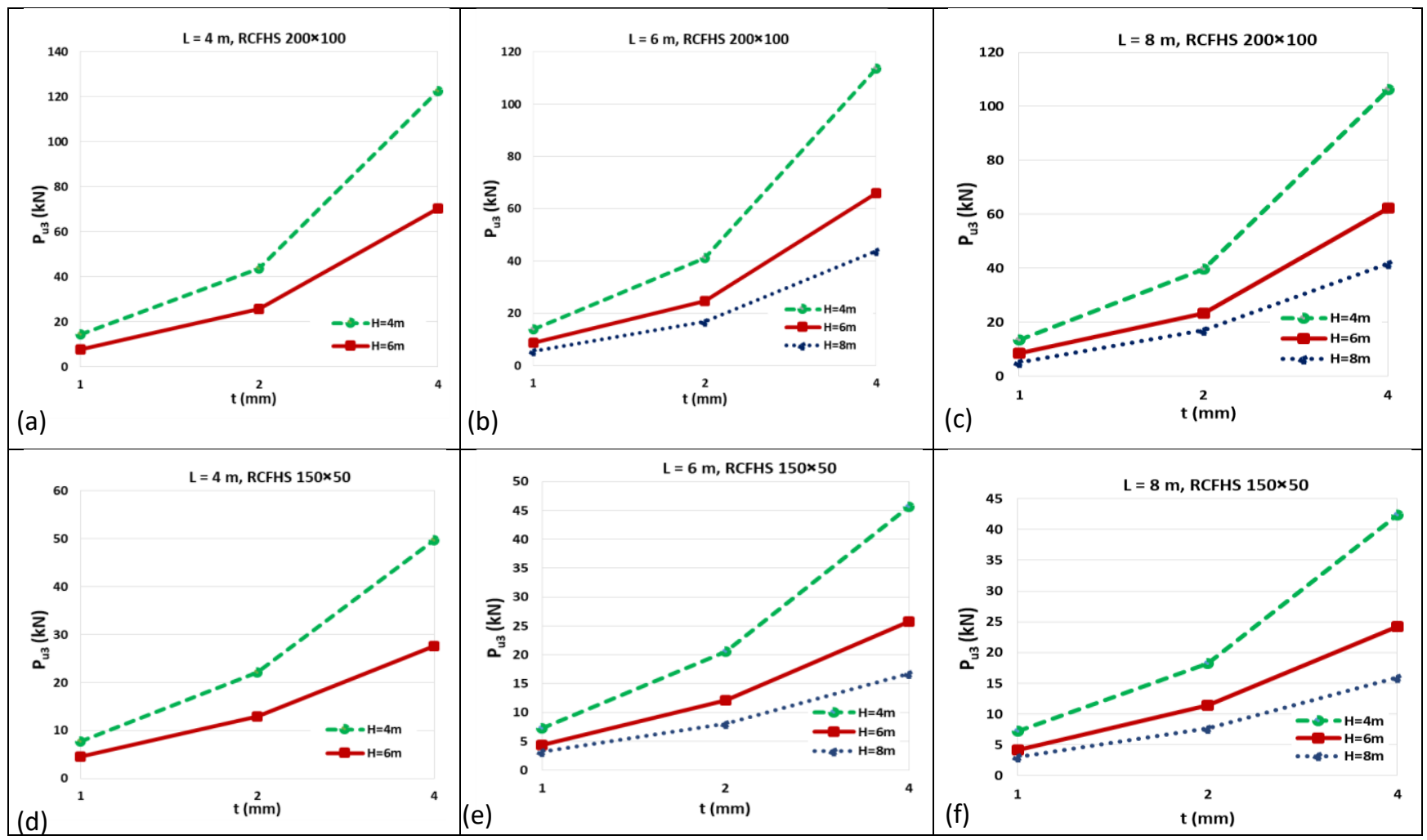

Figure 24: The relation between ultimate loads and different section thicknesses for frames with section RHS $200 \times 100 \mathrm{~mm}$ and

RHS $150 \times 50 \mathrm{~mm}$ using imperfection and residual stresses in analysis (case 3). 


\subsubsection{Failure modes}

All FE models of steel frames failed in-plane instability the out of plane buckling and lateral torsional buckling effects were assumed to be omitted in the analysis. In the presence of residual stresses and imperfection (case 3) in all models of steel frames, different modes of failure appeared. These modes were found in form of material yielding, local buckling and plastic hinges formation. However, some models such as frames with $1 \mathrm{~mm}$ thick sections the failure was mostly due to local buckling although the material yielded at some limited locations, interaction of yielding and local buckling with appearing plastic hinge in some models. But some models with thicknesses 2 and $4 \mathrm{~mm}$, failed due to plastic hinge formed mostly at left beam end and in right column connection as shown in Figure 25.

For example, as shown in Figure 25, the frame 10 with span $6 \mathrm{~m}$, height $6 \mathrm{~m}$, and RHS $200 \times 100 \times 1 \mathrm{~mm}$ is due to local buckling in columns and near beam ends. In addition plastic hinge was formed in the connection of right column whereas the failure in frame 11 with $2 \mathrm{~mm}$ thickness was due to plastic hinge formed at right column connection. Finally, frame 12 with $4 \mathrm{~mm}$ thickness the failure was due to plastic hinge at beam left end and right column connection.

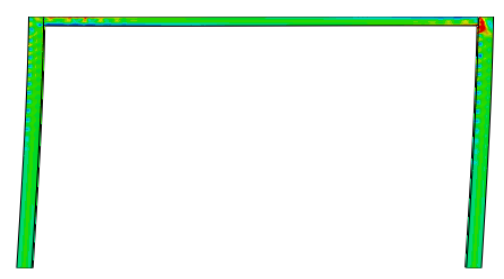

Frame $10, \mathrm{t}=1 \mathrm{~mm}$

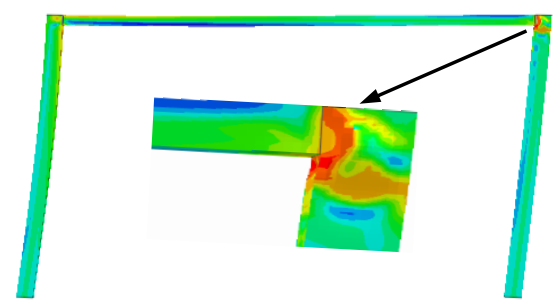

Frame $11, \mathrm{t}=2 \mathrm{~mm}$

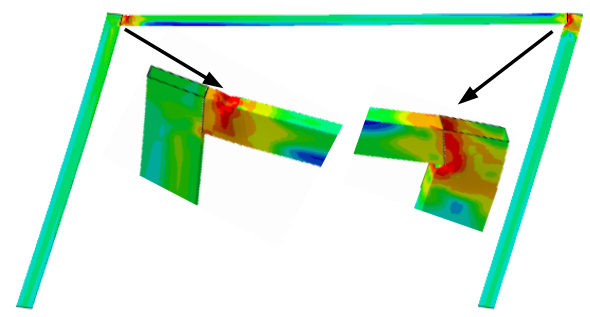

Frame $12, \mathrm{t}=4 \mathrm{~mm}$

Figure 25: Deformed shapes at failure of frames 10, 11 and 12 (case 3)

\section{SUMMARY AND CONCLUSIONS:}

This paper studied numerically the behavior of steel frames fabricated from cold formed rectangular hollow sections using nonlinear finite element analysis. At first, verification was conducted using comparison between the present FEA with four experimental tests from previous studies that were tested to failure under a static loading configuration. The first verification was done for frame tested by Avery [24]. The other three verifications were done for unstiffened, stiffened, and welded sleeve knee joints for cold formed RHS portal frame by Wilkinson and Hancock [29]. An intensive study using FEA of 3D models was carried out to validate the finite element models as well as to detect the effect of presence of initial imperfection and residual stresses. The verification was done through the comparison between FEA and experiments for the load-displacement curves, ultimate strength, and failure modes. Moreover, the method of nonlinear analysis was investigated. The FEA shows good agreement with experimental results thus, the possibility of carrying out a series of nonlinear finite element models increases the efficiency of the analysis even more and enables performing parametric study which are needed. Parametric study was carried out to study the various parameters that affect the behavior of cold formed steel frames. Parameter such as method of imperfection representation, presence of imperfection and residual stresses, cross section size and thickness, and frame dimensions were investigated. The effect of each parameter on the load-displacement curves, ultimate load capacity, maximum horizontal and vertical displacements, and failure modes.

The present study shows that:

4.1 Both static-general based on Newton-Raphson and static-Riks nonlinear analysis methods with load control solution can accurately trace the loaddisplacement curve up to the peak load, but only the static-Riks method can trace the whole loaddisplacement curve till failure.

4.2 The medium mesh density with shell element S4R5 is the most suitable in the present nonlinear FE analysis that it satisfies the fast solution speed and less disk storage, good representation of imperfection, as well as acceptable solution accuracy.

4.3 It is recommended to include both residual stresses and initial imperfections during the nonlinear analysis of cold formed steel frames with rectangular hollow sections.

4.4 The most suitable method of representing the initial imperfection in the nonlinear FEA of whole frame is to use a magnitude of $0.5 \mathrm{t}$ along with the second elastic buckling mode shape. While it is found that during the analysis of knee joints it is suitable to use a value of $0.1 \mathrm{t}$ imperfection with other mode shape numbers. 
4.5 Increasing the thickness of the cross sections has a significant effect on increasing both the ultimate load capacity, horizontal displacement, and vertical mid-span deflection. The overall efficiency of the frame indicated using the percentage ultimate load to weight ratio improved significantly by increasing the cross-section thickness.

4.6 The dimensions of the cross-section affect the value of the ultimate load capacity of the frame that is the dimensions of the cross-section influence both the axial load and bending moment capacities. In addition, the global buckling of the frames can occur faster in frames with smaller dimensions.

4.7 Increasing the frame span has a slight effect on decreasing the ultimate load capacity while increasing the frame height has the greatest effect on decreasing the ultimate load capacity of the frame.

4.8 All the studied parameters have recognizable effect on the failure mode shapes as indicated in the discussion.

\section{Credit Authorship Contribution Statement}

Sara F. Elglaad: Generating the idea, Collecting data, Methodology \& Original draft preparation, Mohamed M. ElGhandour: Reviewing \& Supervision, Ashraf E. ElSabbagh and Tarek A.Sharaf : Validation, Editing, Reviewing \& Supervision.

\section{Declaration of Competing Interest}

The authors declare that they have no known competing financial interests or personal relationships that could have appeared to influence the work reported in this paper.

\section{References}

[1] Androić, B., Dujmović, D., \& Pišković, J. (2014). Application of cold formed hollow steel sections. Građevinar, 66(10.), 929-935

[2] Liu, Z., Liu, H., Chen, Z., \& Zhang, G. (2018). Structural behavior of cold-formed thick-walled rectangular steel columns. Journal of Constructional Steel Research, 147, 277-292.

[3] Dewolf, J. T., Peokoz, T., \& Winter, G. (1974). Local and overall buckling of cold-formed members. Journal of the structural Division, 100(10), 2017-2036.

[4] Zhu, A. Z. (2004). Experimental Investigation of ColdFormed Effect on Thick-Walled Steel Members and Analysis of the Cold-Formed Residual Stress Field (master's degree thesis).

[5] Li, L. M., Jiang, X. L., Chen, Z. H., \& LI, N. (2008). Strain Hardening of Thick-Walled Cold Formed Steel Tube [J]. Journal of Tianjin University, 1.

[6] Wilkinson, T. J. (1999). The plastic behavior of coldformed rectangular hollow sections.

[7] Wilkinson, T., \& Hancock, G. J. (2000). Tests to examine plastic behavior of knee joints in cold-formed RHS. Journal of Structural Engineering, 126(3), 297305

[8] Wilkinson, T., \& Hancock, G. J. (1998). Tests of portal frames in cold-formed RHS. In TUBULAR STRUCTURES-INTERNATIONAL SYMPOSIUM- (Vol. 8, pp. 521-530). E \& FN SPON.
[9] Mang, F., Herion, S., \& Karcher, D. (1997). L-joints made of circular hollow sections. Final report of CIDECT project, (5).

[10] Karcher, D., \& Puthli, R. (2001, January). Design recommendations for stiffened and unstiffened L-joints made of CHS and RHS under fatigue loading. In the Eleventh International Offshore and Polar Engineering Conference. International Society of Offshore and Polar Engineers.

[11] Kwon, Y. B., Chung, H. S., \& Kim, G. D. (2008). Development of cold-formed steel portal frames with PRY sections. Advances in Structural Engineering, 11(6), 633-649

[12] Chung, K. F., \& Lau, L. (1999). Experimental investigation on bolted moment connections among cold formed steel members. Engineering Structures, 21(10), 898-911.

[13] Darcy, G., \& Mahendran, M. (2000). Full Scale Tests of the MGA Building System. Research Report, Physical Infrastructure Centre, Brisbane, Australia.

[14] Kwon, Y. B., Chung, H. S., \& Kim, G. D. (2006). Experiments of cold-formed steel connections and portal frames. Journal of Structural Engineering, 132(4), 600-607.

[15] Kwon, Y. B., Kim, N. G., \& Hancock, G. J. (2007). Compression tests of welded section columns undergoing buckling interaction. Journal of Constructional Steel Research, 63(12), 1590-1602.

[16] Chung, K. F., Ho, H. C., Wang, A. J., \& Yu, W. K. (2008). Advances in analysis and design of cold-formed steel structures. Advances in Structural Engineering, 11(6), 615-632.

[17] Darcy, G., \& Mahendran, M. (2008). Development of a new cold-formed steel building system. Advances in Structural Engineering, 11(6), 661-677.

[18] Puthli, R., Mang, F., \& Karcher, D. (1998). Investigations on stiffened and unstiffened L-joints made of circular hollow sections. In Eighth International Symposium on Tubular Structures, Singapore (Vol. 26, No. 28).

[19] Choo, Y. S., Ren, Y. B., Liew, J. Y. R., \& Puthli, R. S. (2001). Static strength of un-stiffened CHS Knee joint under compression loading. In TUBULAR STRUCTURES-INTERNATIONAL SYMPOSIUM- (Vol. 9, pp. 213-220).

[20] Karcher, D., \& Puthli, R. (2001). The Static Design of stiffened and unstiffened CHS L-joints. In Ninth International Symposium on Tubular Structures (pp. 221-228).

[21] Wilkinson, T., \& Hancock, G. J. (1998). Tests of stiffened and unstiffened welded knee connections in cold-formed RHS. In TUBULAR STRUCTURESINTERNATIONAL SYMPOSIUM- (Vol. 8, pp. 177186). E \& FN SPON.

[22] Wilkinson, T., \& Hancock, G. J. (1998). Tests of bolted and internal sleeve knee connections in cold-formed RHS. A. A. Balkema Publishers, Tubular Structures VIII(USA), 187-195.

[23] Avery, P., \& Mahendran, M. (2000). Distributed plasticity analysis of steel frame structures comprising non-compact sections. Engineering Structures, 22(8), 901-919.

[24] Avery, P. (1998). Advanced analysis of steel frame structures comprising non-compact sections (Doctoral dissertation, Queensland University of Technology).

[25] Baigent, A. H., and Hancock, G. J. (1982). The behavior of portal frames composed of cold-formed members. Thin-walled structures - Recent technical advances and trends in design, research and construction, Elsevier Applied Science, Oxford, 209. 
[26] Kirk, P. (1986). Design of a cold-formed section portal frame building system. Proc., 8th Int. Speciality Conf. on Cold-formed Steel Structures, St. Louis, University of Missouri-Rolla, 295.

[27] De Vos, G. P., \& Van Rensburg, B. W. J. (1997). Lightweight cold-formed portal frames for developing countries. Building and environment, 32(5), 417-425.

[28] Kurobane, Y. (2002). Connections in tubular structures. Progress in Structural Engineering and Materials, 4(1), 35-45.

[29] Wilkinson, T., Ma, B. B., \& Hancock, G. J. (1998). Tests of knee joints in cold-formed rectangular hollow sections.

[30] Schafer, B. W., Li, Z., \& Moen, C. D. (2010). Computational modeling of cold-formed steel. ThinWalled Structures, 48(10-11), 752-762.

[31] Key, B. W., \& Hancock, G. J. (1985). An experimental investigation of the column behaviour of cold formed square hollow sections (No. Rept. No 493).

[32] Packer, J. A., Wardenier, J., Kurobane, Y., Dutta, D., \& Yeomans, N. (1992). Design guide for rectangular hollow section (RHS) joints under predominantly static loading-CIDECT. TíV-Verlag GmbH, Köln, Germany.

[33] Mang, F., Steidl, G., Bucak, O., \& International Institute of Welding: IIW-Doc. XV-463-80. (1980). Design of welded lattice joints and moment resisting knee joints made of hollow sections. Document No. XV-463-80, International Institute of Welding, University of Karlsruhe, Germany

[34] Schafer, B. W., \& Peköz, T. (1998). Computational modeling of cold-formed steel: characterizing geometric imperfections and residual stresses. Journal of constructional steel research, 47(3), 193-210.

[35] Dawson, R. G., \& Walker, A. C. (1972). Post-buckling of geometrically imperfect plates. Journal of the Structural Division, 98(1), 75-94.

[36] Zhu, J. H., \& Young, B. (2011). Cold-formed- steel oval hollow sections under axial compression. Journal of Structural Engineering, 137(7), 719-727.

[37] Key, P. W., (1988), "The Behaviour of Cold-Formed Square Hollow Section Columns", PhD Thesis, School of Civil and Mining Engineering, University of Sydney, Sydney, Australia.

[38] Simulia, ABAQUS Theory Manual: Version 6.12, Simulia, 2012.

[39] EN 10219 (2006): Cold formed welded structural hollow sections of non-alloy and fine grain steels - Part 1: Technical delivery conditions; - Part 2: Tolerances, dimensions and sectional properties.

[40] EL-Ghandour, M. (2009). Development of Cold Formed Knee Joints Constructed of Rectangular Hollow Sections. Ain Shams Journal of Civil Engineering. Vol. 2., pp.95-107

[41] V.Priyadarshini1, Md Hasan Khan2, Murugesan.P3, Kiran Krishna4, Md Riyas5 (2018, mar).STUDY ON THE BEHAVIOUR OF COLD FORMED STEEL FRAMES HOLLOW SECTION. In International Research Journal of Engineering and Technology (IRJET). 